\title{
Ubiquitin-specific protease 7 sustains DNA damage response and promotes cervical carcinogenesis
}

\author{
Dongxue Su, ${ }^{1}$ Shuai Ma, ${ }^{2}$ Lin Shan, ${ }^{1}$ Yue Wang, ${ }^{1}$ Yuejiao Wang, ${ }^{2}$ Cheng Cao, ${ }^{2}$ Beibei Liu, ${ }^{1}$ Chao Yang, ${ }^{1}$ Liyong Wang, ${ }^{3}$ \\ Shanshan Tian, ${ }^{2}$ Xiang Ding, ${ }^{4}$ Xinhua Liu, ${ }^{2} \mathrm{Na}$ Yu, ${ }^{2}$ Nan Song, ${ }^{2}$ Ling Liu, ${ }^{2}$ Shangda Yang, ${ }^{2}$ Qi Zhang, ${ }^{2}$ Fuquan Yang, ${ }^{4}$ \\ Kai Zhang, ${ }^{2}$ and Lei Shi ${ }^{2}$

\begin{abstract}
'Department of Biochemistry and Molecular Biology, School of Basic Medical Sciences, Capital Medical University, Beijing, China. ${ }^{2} 2011$ Collaborative Innovation Center of Tianjin for Medical Epigenetics, Tianjin Key Laboratory of Medical Epigenetics, Key Laboratory of Breast Cancer Prevention and Therapy (Ministry of Education), Key Laboratory of Immune Microenvironment and Disease (Ministry of Education), Department of Biochemistry and Molecular Biology, School of Basic Medical Sciences, Tianjin Medical University Cancer Institute and Hospital, Tianjin Medical University, Tianjin, China. ${ }^{3}$ Core Facilities for Molecular Biology, Capital Medical University, Beijing, China. “'Laboratory of Proteomics, Institute of Biophysics, Chinese Academy of Sciences, Beijing, China.
\end{abstract}

\begin{abstract}
Central to the recognition, signaling, and repair of DNA double-strand breaks (DSBs) are the MRE11-RAD50-NBS1 (MRN) complex and mediator of DNA damage checkpoint protein 1 (MDC1), the interplay of which is essential for initiation and amplification of the DNA damage response (DDR). The intrinsic rule governing the regulation of the function of this molecular machinery remains to be investigated. We report here that the ubiquitin-specific protease USP7 was physically associated with the MRN-MDC1 complex and that the MRN-MDC1 complex acted as a platform for USP7 to efficiently deubiquitinate and stabilize MDC1, thereby sustaining the DDR. Accordingly, depletion of USP7 impaired the engagement of the MRN-MDC1 complex and the consequent recruitment of the downstream factors p53-binding protein 1 (53BP1) and breast cancer protein 1 (BRCA1) at DNA lesions. Significantly, USP7 was overexpressed in cervical cancer, and the level of its expression positively correlated with that of MDC1 and worse survival rates for patients with cervical cancer. We demonstrate that USP7-mediated MDC1 stabilization promoted cervical cancer cell survival and conferred cellular resistance to genotoxic insults. Together, our study reveals a role for USP7 in regulating the function of the MRN-MDC1 complex and activity of the DDR, supporting the pursuit of USP7 as a potential therapeutic target for MDC1-proficient cancers.
\end{abstract}

\section{Introduction}

To ensure genome integrity, eukaryotic cells are equipped with sophisticated molecular machinery to repair various DNA lesions generated by genotoxic insults from intrinsic and extrinsic sources (1). DNA double-strand breaks (DSBs) are the most cytotoxic type of DNA damage, as unrepaired or inappropriate repair of DSBs will inevitably cause mutations or chromosomal aberrations and eventually lead to diseases such as cancer (2-4). Therefore, the ability of cells to maintain genomic stability through a temporally and spatially orchestrated DNA damage response (DDR) is vital for cellular homeostasis.

Among the first regulators that initially accumulate at sites of DSBs in eukaryotic cells is the MRE11-RAD50-NBS1 (MRN) complex, a conserved and essential DDR factor that controls DSB repair, checkpoint signaling, and meiotic recombination (5, 6). Specifically, MRE11 is a structure-specific nuclease involved in the resection of DNA ends to allow homologous recombination (HR) repair, while RAD50 bears both ATPase and adenylate kinase activities, together with MRE11, facilitating binding of the MRN complex to DNA molecules and thus DSB repair (7-9). The third subunit of the MRN complex, NBS1, belongs to the family of

Authorship note: DS and SM contributed equally to this work. Conflict of interest: The authors have declared that no conflict of interest exists. Submitted: February 14, 2018; Accepted: July 24, 2018.

Reference information: J Clin Invest. 2018;128(10):4280-4296.

https://doi.org/10.1172/JCl120518. adaptor and mediator proteins that integrate and coordinate the DDR through the promotion of protein-protein interactions (10, 11). Another core component in initial DDR signaling is the mediator of DNA damage checkpoint protein 1 (MDC1), which interacts with the MRN complex prior to the detection and signaling of DNA damage (12-14).

In response to DNA damage, the MRN complex recognizes DSBs and recruits the central DDR signaling kinase ataxiatelangiectasia mutated (ATM) to phosphorylate histone H2AX $(5,15)$. Subsequently, MDC1 binds to phosphorylated H2AX $(\mathrm{p}-\gamma \mathrm{H} 2 \mathrm{AX})$ and facilitates further accumulation and retention of the MRN complex as well as the E3 ligases RNF8 and RNF168 to promote the formation of lysine 63-linked (K63-linked) polyubiquitin chains on chromatin and the chromatin binding of p53binding protein 1 (53BP1) and breast cancer protein 1 (BRCA1) (14, 16-20), generating microscopically discernible nuclear foci for efficient repair. Although it is well established that, in damaged chromatin regions, the $\gamma \mathrm{H} 2 \mathrm{AX}-\mathrm{MDC} 1$ binding module further promotes the recruitment of the MRN complex and thus ATM to amplify and sustain the damage-sensing and repair signal $(5,21)$, it is still unclear how this positive feedback loop dictated by MDC1 and the MRN complex is achieved and regulated.

Deficiency or mutation of key components of the DDR predisposes individuals to hereditary cancer (22-25). As such, DDR activation is considered an inducible barrier to tumorigenesis. Nevertheless, cancerous cells often carry abnormalities in the DSB repair machinery and adjust their DDR programs to deal with 
DNA damage loads associated with excessive replication, which is essential for the survival of these cells and renders cells resistant to DNA damage-based therapy $(1,26,27)$. For example, deregulation of the key players involved in DSB repair is believed to play an important role in the development and progression of cervical cancer, the fourth most common cause of malignancy and deaths from cancer in women worldwide $(28,29)$. Specifically, a high level of ATM activation is associated with resistance to ionizing radiation and disease-specific survival (30), and increased copy numbers of ataxia-telangiectasia and Rad3-related (ATR) protein have also been implicated in the pathogenesis of cervical cancer (31). However, the mechanistic basis and pathological relevance of the dysregulation of the DDR in cervical carcinogenesis remain to be investigated.

Accumulating evidence suggests that a multitude of posttranslational protein modifications are involved in the DDR process (32-35). In particular, ubiquitination is emerging as a central player in DSB repairs (17-20,36). Ubiquitination is constantly opposed through the action of specific deubiquitinating enzymes (DUBs), of which ubiquitin-specific proteases (USPs) comprise the largest subfamily (37). Among all USPs, USP7, also known as herpesvirus-associated ubiquitin-specific protease (HAUSP) (38), has attracted the most attention because of its involvement in multiple oncogenic pathways (39). Interestingly, USP7 has been reported to target a panel of DDR proteins, including RNF168 (40), RNF169 (41), CHK1 (42, 43), and claspin (44). Despite this, it remains an open question as to how USP7 contributes to the response to and repair of DSBs, especially at the initial step of the DDR.

In this study, we report that USP7 is physically associated with the MRN-MDC1 complex and recruited to the damage site of DSBs in an MRN-MDC1 complex-dependant manner. We show that USP7 facilitates DNA repair by stabilizing MDC1, thereby sustaining the MRN-MDC1 complex around DSBs. Importantly, we found that USP7-promoted deubiquitination and stabilization of MDC1 is implicated in cervical carcinogenesis and confers a resistance of cervical cancer cells to genotoxic insults.

\section{Results}

USP7 is physically associated with the MRN-MDC1 complex. To further understand how the MRN-MDC1 complex coordinates the response to and repair of DSBs, we used affinity purification and mass spectrometry to interrogate the interactomes of MRE11, NBS1, and MDC1 in vivo. Interestingly, the results indicated that the ubiquitin-specific protease USP7, among other proteins, was efficiently copurified with each of these 3 components of the MRN-MDC1 complex (Figure 1A and Supplemental File 1). To confirm the in vivo association of USP7 with the MRN-MDC1 complex, we performed co-IP experiments. The results showed that USP7 was coimmunoprecipitated with each component of the MRN-MDC1 complex, and vice versa (Figure 1B). Notably, the association of USP7 with the MRN-MDC1 complex was independent of DNA incorporation, since DNase treatment showed a marginal effect on the integrity of the whole complex (Supplemental Figure 1A; supplemental material available online with this article; https://doi.org/10.1172/JCI120518DS1).

To further validate the in vivo interaction between USP7 and the MRN-MDC1 complex, we performed protein fractionation experiments by fast protein liquid chromatography (FPLC) using a Superose 6 column and a size-exclusion approach. The results indicated that native USP7 from HeLa cells was eluted with an apparent molecular mass much greater than that of the monomeric protein, and we detected USP7 in a large molecular mass peak that coeluted with the MRN-MDC1 complex in fractions 17 to 21 (Figure 1C). Furthermore, analysis of the FLAG-USP7 affinity eluate by FPLC with Superose 6 gel filtration revealed that the majority of purified FLAG-USP7 existed in a multiprotein complex, which peaked in fractions 17 to 21 containing the MRNMDC1 complex (Figure 1D). Analogously, gel filtration analysis of the FLAG-MDC1 affinity eluate showed that USP7 and the MRN complex were copurified with MDC1 in fractions 17 to 19 (Supplemental Figure 1B). In addition, immunofluorescence staining followed by confocal microscopic analysis revealed that endogenous USP7 appeared to be colocalized with all components of the MRNMDC1 complex in the nucleus of HeLa cells (Figure 1E).

To further support the interaction between USP7 and the MRN-MDC1 complex and to gain molecular insights into this interaction, we generated GFP-tagged domain deletion mutants of USP7 and transfected them into HeLa cells. Immunoprecipitation (IP) with anti-GFP followed by immunoblotting (IB) with antibodies against MDC1 indicated that the ubiquitin-like (UBL) domain in the C terminus of USP7 was responsible for the interaction of USP7 with MDC1 (Supplemental Figure 1C). Reciprocally, domain mapping of the molecular interface of MDC1 required for USP7 binding revealed that the large central proline-serinethreonine-rich (PST-rich) repeats domain was required for the association of MDC1 with USP7 (Supplemental Figure 1D). Moreover, glutathione S-transferase-pulldown (GST-pulldown) experiments with GST-fused PST or the BRCA1 C terminus (BRCT) domain of MDC1 and full-length or truncations of the recombinant USP7 confirmed that the PST domain of MDC1 specifically interacts with the UBL domain of USP7 (Supplemental Figure 1E). Additionally, pulldown experiments with full-length recombinant USP7 and in vitro-transcribed and -translated MRE11, RAD50, or NBS1 revealed that USP7 directly interacted with NBS1 (Supplemental Figure 1F, left), and domain mapping analysis with recombinant proteins further indicated that the meprin and TNF receptor-associated factor (TRAF) homology (MATH) domains in the N-terminus of USP7 and the C-terminal unstructured region of NBS1 were responsible for the association of these 2 molecules (Supplemental Figure 1F, right; Supplemental Figure 1G). Collectively, these results suggest that USP7 directly interacts with the MRN-MDC1 complex through distinct molecular surfaces (Supplemental Figure 1H).

USP7 is recruited to damage sites in an MRN-MDC1dependent manner. The physical association of USP7 with the MRN-MDC1 complex implies a role for USP7 in the cellular response to DNA damage. To test this, we first investigated whether USP7 is recruited to DSBs using an endonuclease AsiSI-based system, in which endogenous sequence-specific DSBs could be generated in the presence of 4-hydroxyltamoxifen (4-OHT) (45). Quantitative ChIP (qChIP) analysis revealed that, like $\gamma \mathrm{H} 2 \mathrm{AX}$, USP7 was enriched around the break proximal site, but not the distal region approximately $2 \mathrm{Mb}$ away from the break site upon AsiSI activation (Figure 2A). In addition, 
A

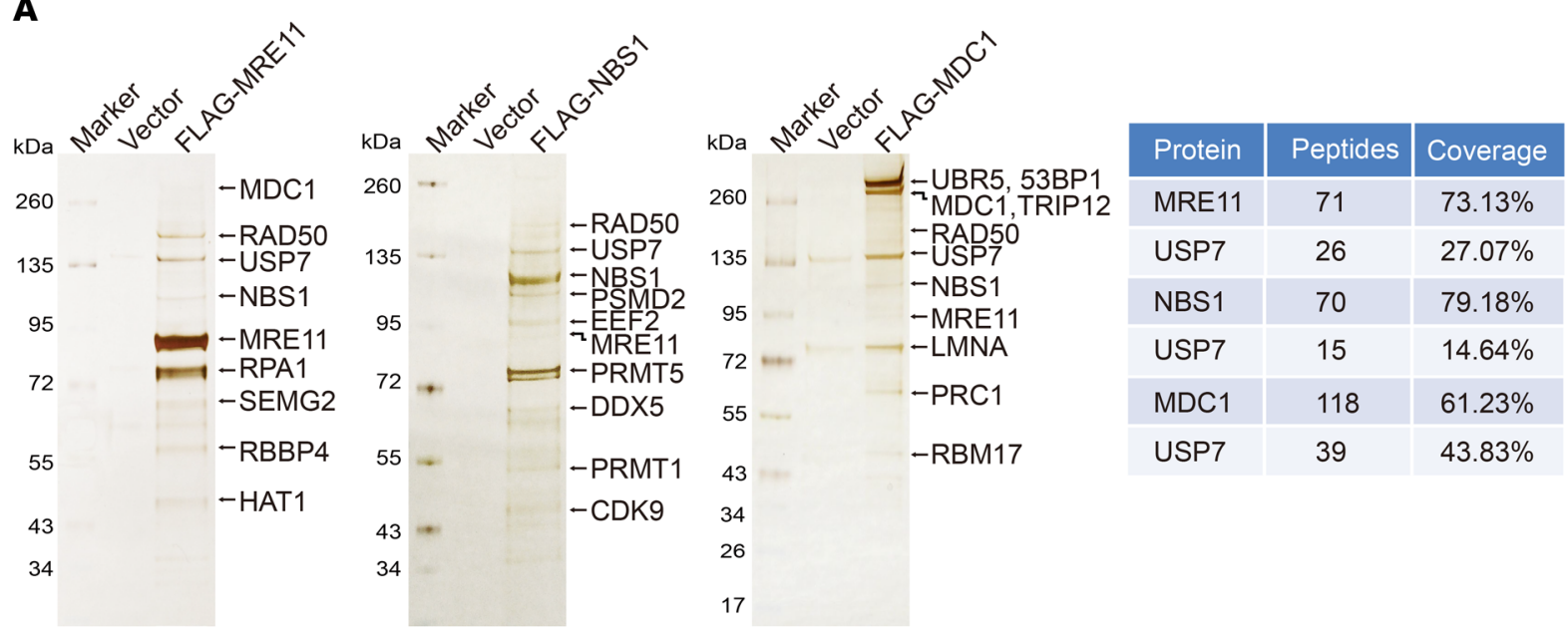

B
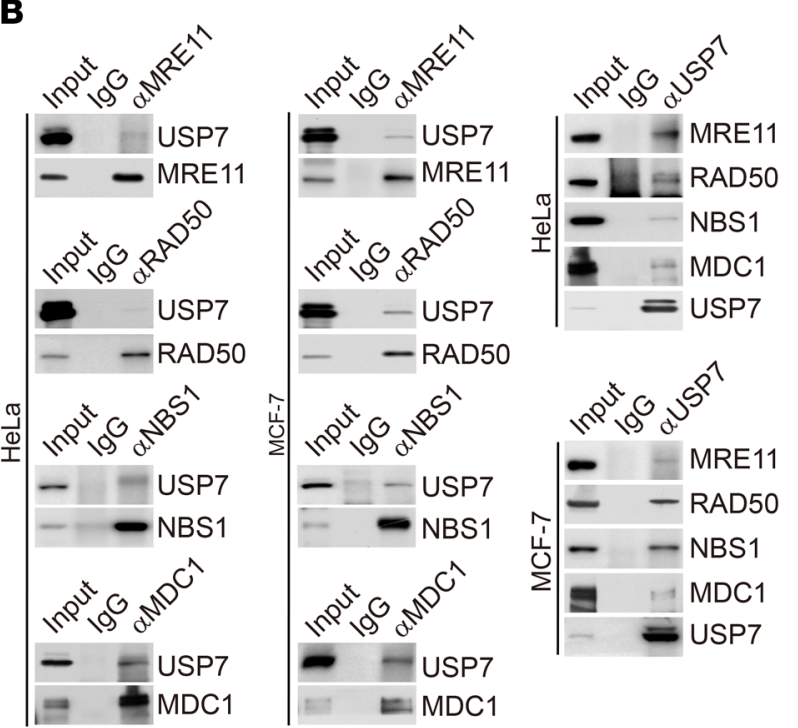

C
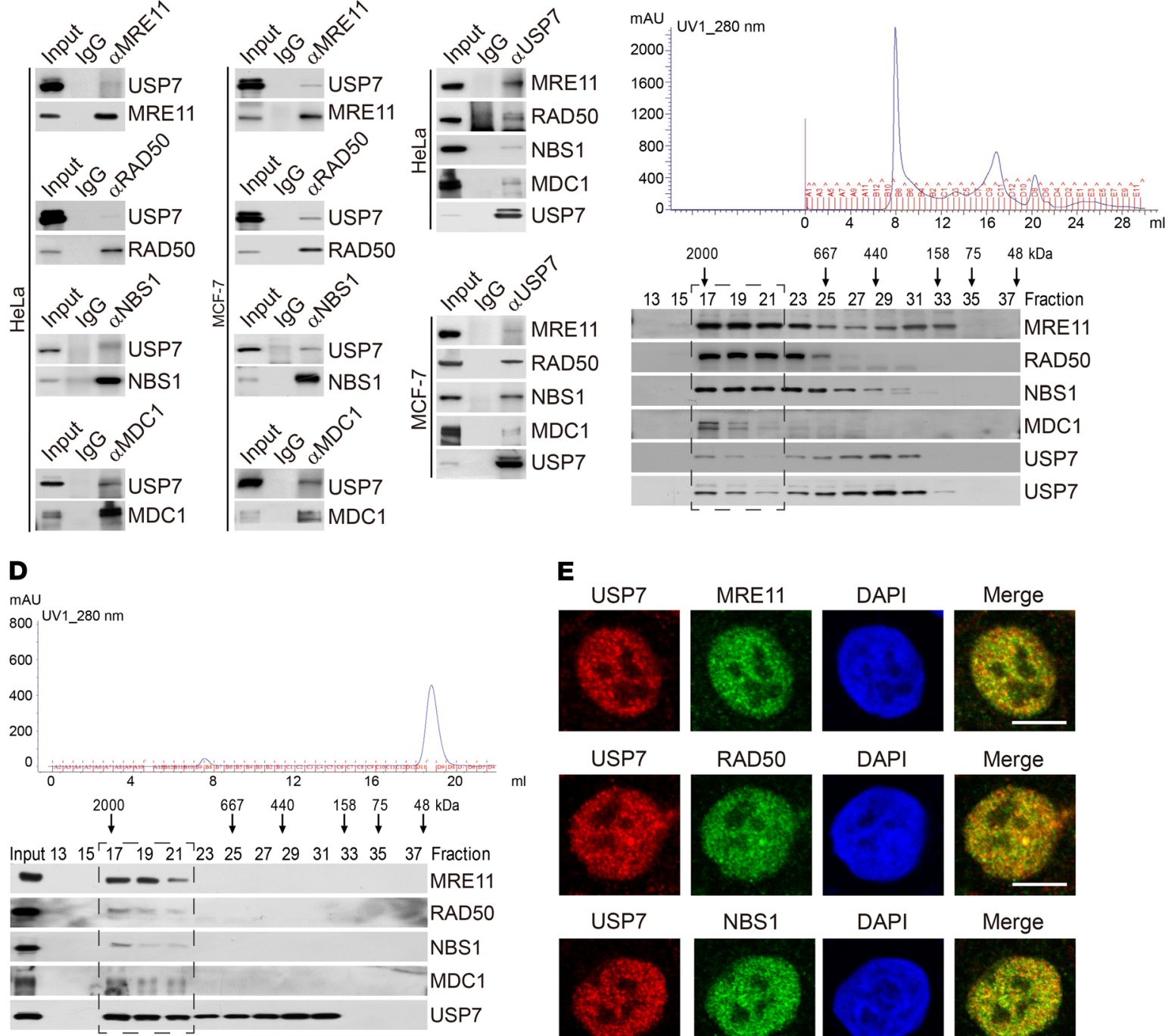

E
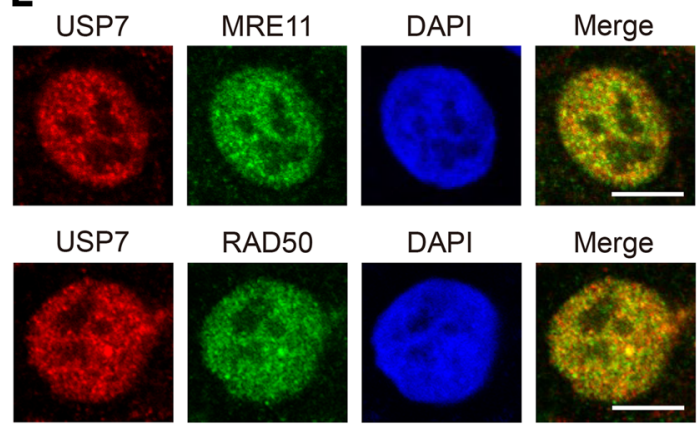

Merge
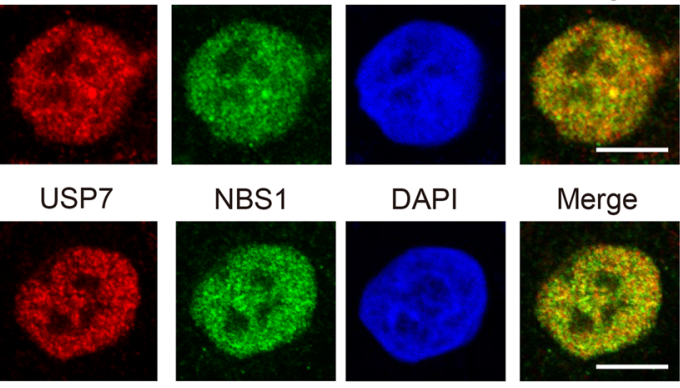

Merge
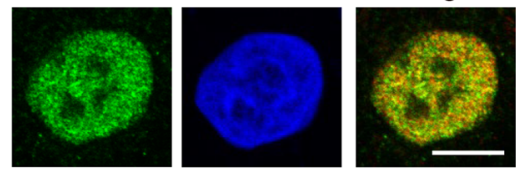

USP7

MDC1

DAPI

Merge
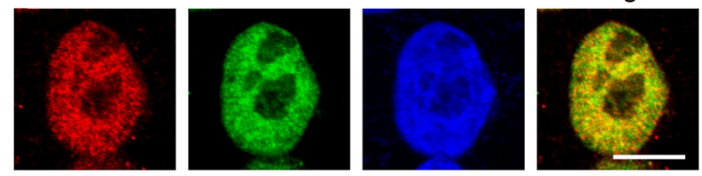
Figure 1. USP7 is physically associated with the MRN-MDC1 complex. (A) Immunopurification and mass spectrometric analysis of MRE11-, NBS1-, or MDC1-containing protein complexes. Cellular extracts from HeLa cells stably expressing FLAG-MRE11, FLAG-NBS1, or FLAG-MDC1 were immunopurified with anti-FLAG affinity beads and eluted with FLAG peptide. The eluates were resolved on SDS/PAGE and silver stained, followed by mass spectrometric analysis. The percentage of peptide coverage of the indicated proteins is shown. (B) Co-IP analysis of the association between USP7 and the MRN-MDC1 complex. Whole-cell lysates from HeLa and MCF-7 cells were immunoprecipitated and then immunoblotted with antibodies against the indicated proteins. $\alpha$, anti-. (C) FPLC analysis of the native protein complex. Nuclear extracts from HeLa cells were fractionated on Superose 6 sizeexclusion columns with high-salt buffer. Chromatographic elution profiles and Western blot analysis of the chromatographic fractions with antibodies against the indicated proteins are shown. Equal volumes from each fraction were analyzed, and the elution positions of the calibration proteins with known molecular masses $(\mathrm{kDa})$ are indicated. The boxed area indicates fractions in which endogenous USP7 was coeluted with the MRN-MDC1 complex. (D) Experiments analogous to those for $\mathbf{C}$ were performed with a USP7-containing protein complex purified from FLAG-USP7-expressing HeLa cells. The boxed area indicates fractions in which FLAG-USP7 was coeluted with the MRN-MDC1 complex. (E) Confocal microscopic analysis of USP7 and MRNMDC1 complex subcellular localization. HeLa cells were fixed and immunostained with antibodies against the indicated proteins. Representative images from biological triplicate experiments are shown. Scale bars: $10 \mu \mathrm{m}$.

USP7 formed discernible, albeit weak, foci at sites of DSBs generated by 4-OHT-driven AsiSI endonuclease, and the majority of these foci were colocalized with MDC1 foci (Figure 2B, top). Meanwhile, a higher dose of $\mathrm{x}$-ray irradiation (IR) also induced foci formation by USP7, and these foci were colocalized with $\gamma \mathrm{H} 2 \mathrm{AX}$ (Figure 2B, bottom). Additionally, confocal microscopy and live-cell imaging analysis showed that GFP-tagged USP7 was recruited to damaged sites rapidly after laser micro-irradiation (micro-IR), manifested by fast accumulation of GFP signal around the break sites (Figure 2C and Supplemental Figure 2A). Furthermore, immunofluorescence staining followed by confocal microscopic analysis indicated that endogenous USP7 was accumulated and colocalized with $\gamma \mathrm{H} 2 \mathrm{AX}$ and MDC1 at UVA laser microdissection-induced DNA lesions (Figure 2D and Supplemental Figure 2B). Together, these results suggest that USP7 is mobilized and recruited to DSB sites upon DNA damage.

To examine whether the involvement of USP7 in DSBs is through its association with the MRN-MDC1 complex, native HeLa cells or HeLa cells stably expressing GFP-USP7 were transfected with control siRNA or siRNA targeting MRE11, RAD50, NBS1, or MDC1. Microscopic analysis showed that DNA damage-induced recruitment of either endogenous USP7 (Figure 2E and Supplemental Figure 2C) or GFP-USP7 (Figure 2F and Supplemental Figure 2D) was severely impaired in cells deficient in each component of the MRN-MDC1 complex, while the expression of endogenous USP7 or GFP-USP7 was essentially unchanged (Supplemental Figure 2, E and F), suggesting that USP7 is recruited to DNA damage sites in an MRN-MDC1 complex-dependent manner. In support of this, examination of the distribution of nuclear localized GFP-tagged USP7 deletion mutants demonstrated that either MATH or UBL, which is responsible for the association of USP7 with NBS1 or MDC1, respectively, could accumulate at DNA damage sites, although with lower efficiency than WT USP7 (Supplemental Figure 2G). Additionally, we also examined the effect of 53BP1, BRCA1, or RNF168 depletion on the recruitment of USP7. Microscopic analysis revealed that loss of function of any of these factors had a marginal effect on laser-induced USP7 stripe formation, indicating that USP7 acts upstream of these repair proteins (Supplemental Figure 3, A-D). Collectively, these data support the notion that the recruitment of USP7 to DNA lesions occurs through its interaction with the MRN-MDC1 complex.

USP7 is functionally linked to the stability of MDC1. To explore the functional significance of the physical interaction and spatial colocalization of USP7 and the MRN-MDC1 complex, we first examined the influence of USP7 on expression of the components of the MRN-MDC1 complex. Western blot analysis showed that knockdown of USP7 in MCF-7 cells had little effect on the protein levels of MRE11, RAD50, NBS1, BRCA1, and 53BP1 (Figure $3 A)$. However, depletion of USP7 resulted in a marked decrease in MDC1 protein levels (Figure 3A). We made a similar observation in HeLa cells (Figure 3B). Meanwhile, quantitative reverse transcription PCR (qRT-PCR) analysis indicated that MDC1 mRNA expression was not affected upon USP7 depletion (Figure 3, A and $\mathrm{B})$. Moreover, KO of USP7 using the CRISPR/Cas9 system revealed that the protein, but not mRNA, levels of MDC1 were significantly reduced (Figure 3C), and this effect could be reversed by overexpression of full-length, but not UBL domain-deleted (USP7/ $\triangle U B L$ ), USP7 (Figure 3D). These observations indicate that MDC1 is a potential substrate of USP7.

To gain further support for the observation that MDC1 is a substrate of USP7, we performed cycloheximide (CHX) chase assays and found that USP7 depletion was associated with a decreased MDC1 half-life (Figure 3E). Moreover, the reduction in MDC1 protein levels associated with USP7 depletion was probably a result of proteasome-mediated protein degradation, as the effect could be effectively blocked by the proteasome-specific inhibitor MG132 (Figure 3F).

Since the MRN complex also directly associates with USP7 through the molecular surface of NBS1 and the MATH domain of USP7, we wondered whether this interaction has an impact on USP7-promoted MDC1 stabilization. We found that knockdown of NBS1 not only impaired the association of USP7 with MDC1 (Figure 3G), but also led to a measurable decrease in MDC1 protein levels (Figure $3 \mathrm{H}$ ), suggesting that the integrity of the MRNMDC1 complex is required for USP7-promoted MDC1 stabilization. Furthermore, we showed that, in USP7-KO cells, full-length, but not MATH domain-deleted (USP7/DMATH), USP7 could efficiently restore MDC1 levels (Figure 3I). Consistently, co-IP analysis revealed that only a minimal amount of MDC1 could be coimmunoprecipitated by USP7/ $\triangle \mathrm{MATH}$ (Figure 3J), although this mutant showed higher affinity for MDC1 than did USP7/ $\triangle \mathrm{UBL}$ (Figure 3J). These observations support the notion that the association of USP7 with the MRN complex, in particular, with NBS1, is functionally linked to the control of MDC1 stability.

USP7 deubiquitinates MDC1. To determine whether USP7promoted MDC1 stabilization is dependent on the enzymatic activity of USP7, we created 2 stable HeLa cell lines with doxycycline-inducible (Dox-inducible) expression of WT USP7 (USP7/ WT) and the catalytically inactive mutant of USP7 (USP7/C223S) (46), respectively. Western blot analysis showed that downregulation of MDC1 under USP7-depleted conditions could be reverted 
A

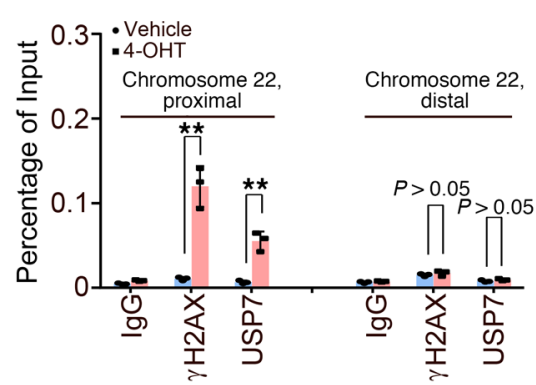

B

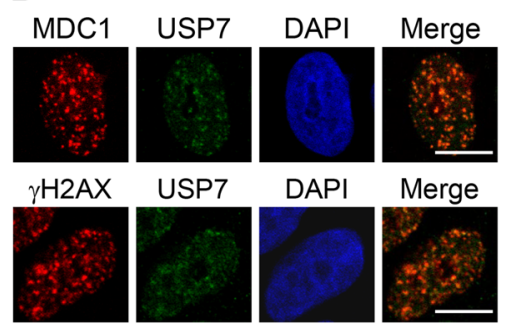

C

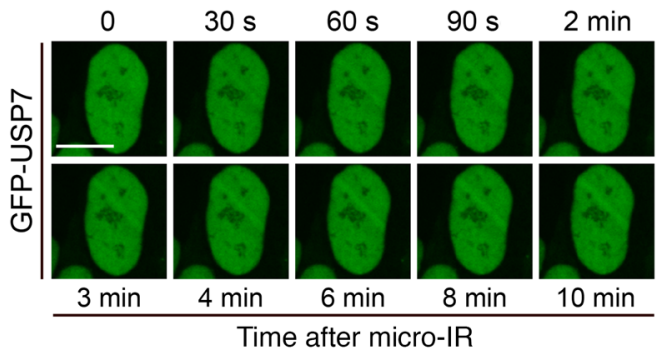

D

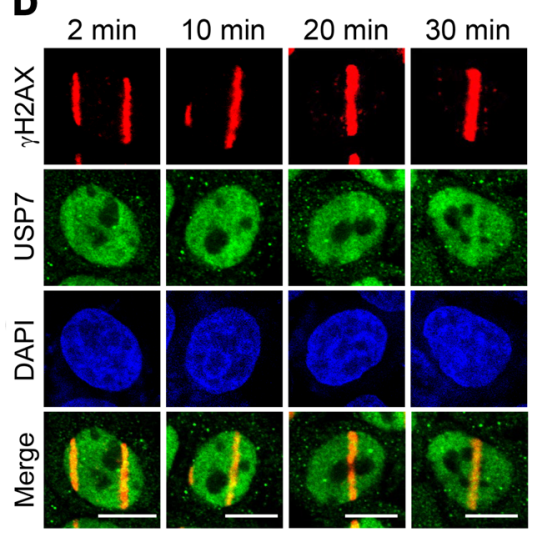

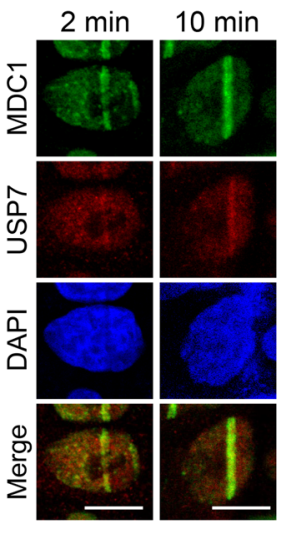

E

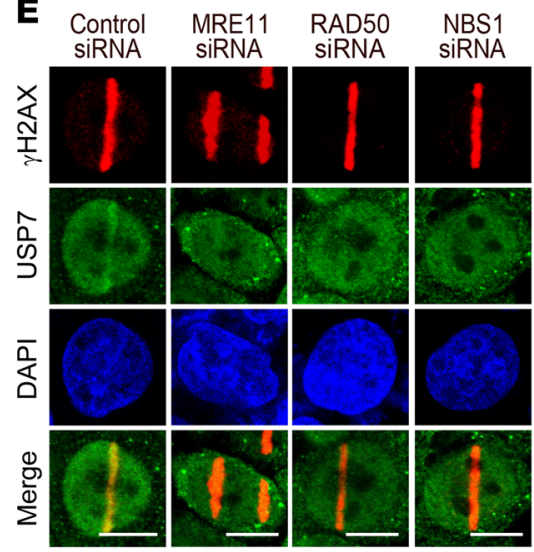

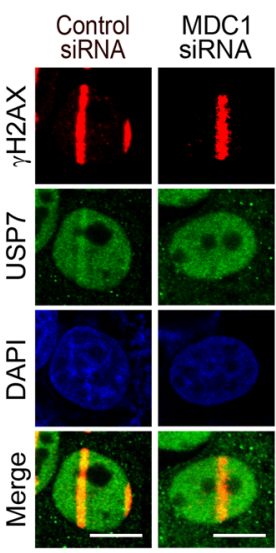

$\mathbf{F}$

Time after micro-IR

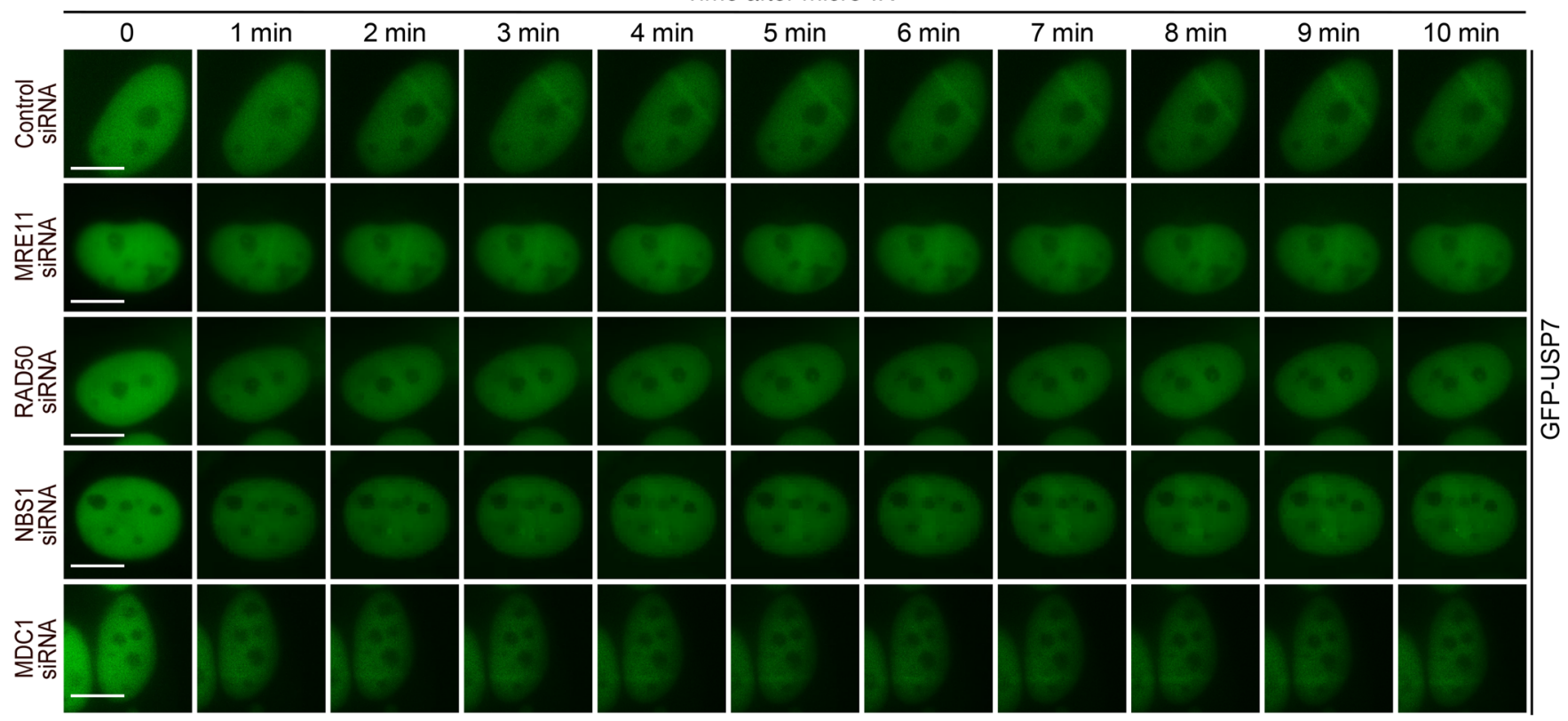

Figure 2. USP7 is recruited to DSB sites. (A) qChIP analysis of USP7 recruitment around sites of DSBs. HeLa cells stably expressing HA-ER-AsiSI were cultured in the absence or presence of $0.5 \mu \mathrm{M} 4-\mathrm{OHT}$. qChIP experiments were performed using anti-USP7 or anti- $\gamma \mathrm{H} 2 \mathrm{AX}$ antibodies with primers that covered the DNA sequences flanking the AsiSI cutting site and the distal region of the break. $\gamma \mathrm{H} 2 \mathrm{AX}$ was used as a positive control. Data represent the mean \pm SD of biological triplicate experiments. ${ }^{* *} P<0.01$, by 2-tailed unpaired Student's $t$ test. (B) Confocal microscopic analysis of USP7 foci formation upon DSBs. Cells used in $\mathbf{A}$ (top) or U2OS cells under x-ray-induced IR (10 Gy, bottom) were preextracted with CSK buffer and then fixed and immunostained with antibodies against USP7 and MDC1 or $\gamma$ H2AX. Scale bars: $10 \mu \mathrm{m}$. (C) HeLa cells stably expressing GFP-USP7 were subjected to laser micro-IR and live-cell imaging at the indicated time points. Scale bar: $10 \mu \mathrm{m}$. (D) HeLa cells subjected to UVA laser microdissection were fixed and immunostained with antibodies against USP7 and $\gamma \mathrm{H} 2 \mathrm{AX}$ or USP7 and MDC1, followed by confocal microscopic analysis. Images are higher-magnification cropped versions of those shown in Supplemental Figure 2B. Scale bars: $10 \mu \mathrm{m}$. (E) HeLa cells were transfected with the indicated siRNAs and subjected to UVA laser microdissection. Then, cells were fixed and immunostained with antibodies against $\gamma \mathrm{H} 2 \mathrm{AX}$ or USP7, followed by confocal microscopic analysis. Images are higher-magnification cropped versions of those shown in Supplemental Figure 2C. Scale bars: $10 \mu \mathrm{m}$. (F) HeLa cells stably expressing GFP-USP7 were transfected with the indicated siRNAs, and cells were then subjected to laser micro-IR and live-cell imaging. Scale bars: $10 \mu \mathrm{m}$. (B-F) Representative images from biological triplicate experiments are shown. 
A

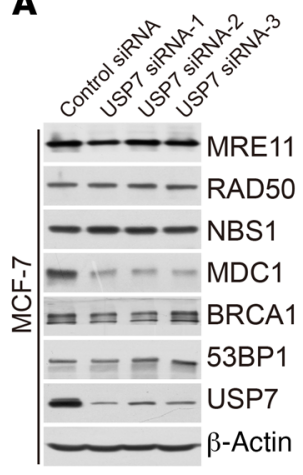

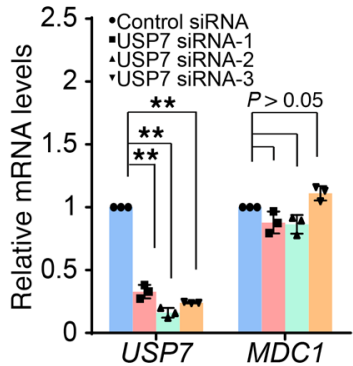

D

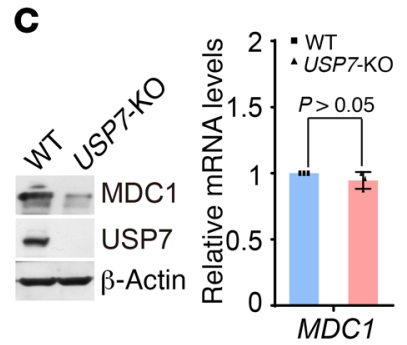

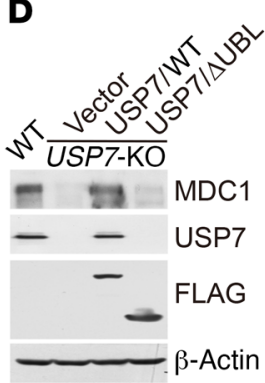

B
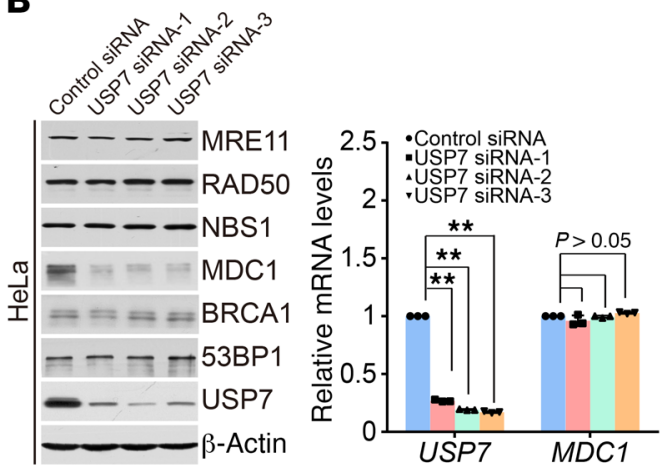

E

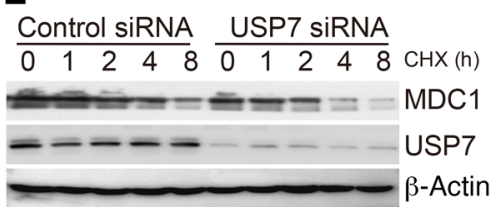

$\mathbf{F}$

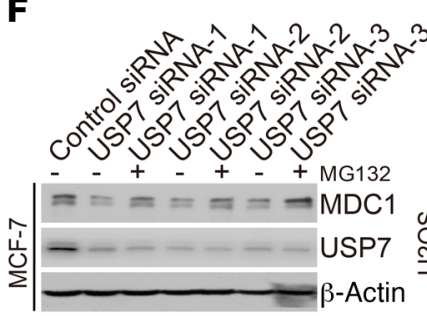

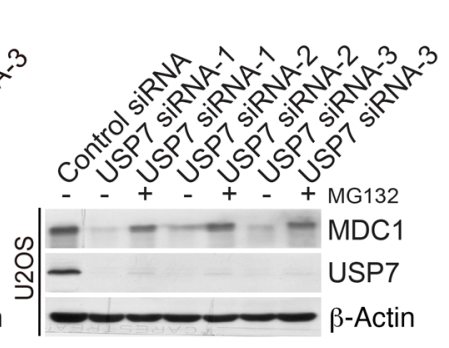
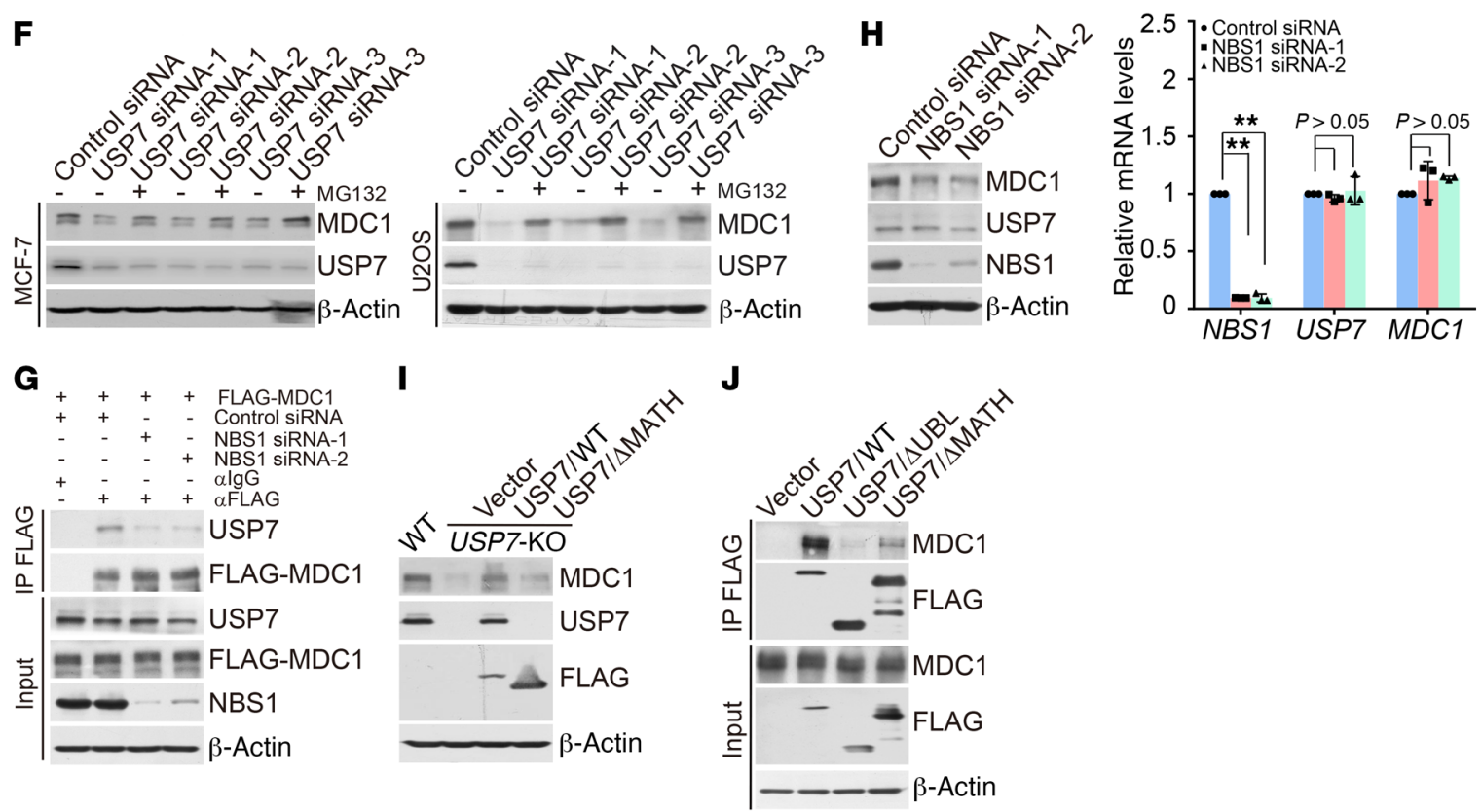

Figure 3. USP7 promotes MDC1 stabilization. (A) MCF-7 cells transfected with control siRNA or different sets of USP7 siRNAs were collected and analyzed by Western blotting and qRT-PCR, respectively. (B) Experiments analogous to the data in A were performed using HeLa cells. (C) Cellular lysates or total RNA from WT and USP7-KO HeLa cells were analyzed by Western blotting and qRT-PCR, respectively. (D) USP7-KO HeLa cells were transfected with control vector, WT USP7 (USP7/WT), or UBL domain-deficient USP7 (USP7/ $\triangle \mathrm{UBL}$ ) cells. Cellular extracts were prepared and analyzed by Western blotting. (E) MCF-7 cells were transfected with control siRNA or USP7 siRNA, followed by treatment with CHX ( $50 \mu \mathrm{g} / \mathrm{ml})$, and harvested at the indicated time points, followed by Western blotting. (F) MCF-7 cells (left) or U20S cells (right) transfected with control siRNA or USP7 siRNAs were treated with DMSO or the proteasome inhibitor MG132 $(10 \mu \mathrm{M})$. Cellular extracts were prepared and analyzed by Western blotting. (G) HeLa cells stably expressing FLAG-MDC1 were transfected with control siRNA or NBS1 siRNAs, and whole-cell lysates were collected for co-IP analysis. (H) HeLa cells transfected with control siRNA or different sets of NBS1 siRNAs were collected and analyzed by Western blotting and qRT-PCR, respectively. (I) USP7-KO HeLa cells were transfected with control vector, USP7/WT, or MATH domain-deficient USP7 (USP7/ $\triangle M A T H)$. Cellular extracts were prepared and analyzed by Western blotting. (J) HeLa cells stably expressing GFP-MDC1 were transfected with FLAG-tagged USP7/WT, USP7/ $U$ UBL, or USP7/ $\triangle M A T H$. Whole-cell lysates were collected for co-IP analysis. In $\mathbf{A}-\mathbf{C}$ and $\mathbf{H}$, data represent the mean $\pm \mathrm{SD}$ of biological triplicate experiments. ${ }^{*} P<0.01$, by 1 -way ANOVA $(\mathbf{A}, \mathbf{B}$ and $\mathbf{H})$ and 2 -tailed unpaired Student's $t$ test (C). 
by forced expression of USP7/WT, but not USP7/C223S (Figure 4A). Moreover, treatment of cells with HBX 41,108, a deubiquitinase inhibitor reported to inhibit USP7 at low micromolar concentrations (47), resulted in a dose-dependent reduction in the protein, but not mRNA, levels of MDC1 (Figure 4B). We obtained similar results with the recently developed USP7 inhibitors GNE6640 and GNE-6776 (Supplemental Figure 4, A and B), which were reported to inhibit USP7 with greater efficacy (48). Together, these results indicate that USP7 regulates the stability of MDC1 through its deubiquitinase activity.

Given our observation that USP7 promotes the stabilization of MDC1 and the report that MDC1 is a ubiquitinated protein (49), we next asked whether USP7 functions to deubiquitinate MDC1. To this end, HeLa cells stably expressing FLAG-MDC1 were cotransfected with control siRNA or different sets of USP7 siRNAs, together with HA-tagged WT ubiquitin (Ub/WT). IP of cellular lysates with anti-FLAG followed by IB with anti-HA showed that knockdown of USP7 resulted in increased levels of ubiquitinated MDC1 species (Figure 4C). Meanwhile, treatment of HeLa cells with USP7 inhibitors led to a marked increase in the level of ubiquitinated MDC1 species (Figure 4D and Supplemental Figure 4C). Moreover, cotransfection of HeLa cells stably expressing FLAG-MDC1 with different amounts of USP7/WT or USP7/C223S together with HA-Ub or a ubiquitin mutant (Ub/mt) with all lysine residues replaced by arginine (50), followed by IP of cellular lysates with anti-FLAG and IB with anti-HA, showed that the levels of ubiquitinated MDC1 species decreased in USP7/WTexpressing cells (Figure 4E), but not USP7/C223S-expressing cells (Figure 4F). Furthermore, we found that USP7/ $\triangle \mathrm{UBL}$ failed to remove polyubiquitin conjugates on MDC1, while USP7/ $\triangle M A T H$ had only a moderate effect on the levels of ubiquitinated MDC1 species (Figure 4G), supporting the idea that the molecular interfaces involved in USP7 binding to the MRN-MDC1 complex act as a platform for USP7 to efficiently deubiquitinate MDC1.

Then, we used a ubiquitin mutant with all lysine residues replaced by arginine except K48 (K48-only) or K63 (K63-only) to differentiate ubiquitin species opposed by USP7 on polyubiquitinated MDC1. The results indicated that K48-linked ubiquitin species conjugated on MDC1 were the major form of ubiquitin moieties opposed by USP7 (Figure $4 \mathrm{H}$ ). In addition, in vitro deubiquitination assays with HA-Ub-conjugated FLAG-MDC1 purified from HeLa cells using high-salt and detergent buffer and His-USP7/WT or His-USP7/C223S purified from Sf9 cells revealed that USP7/WT was capable of deubiquitinating MDC1, whereas USP7/C223S was not (Figure 4I). According to the molecular organization mode of the whole complex, we deduced that addition of the MRN proteins to this in vitro system might promote USP7catalyzed MDC1 deubiquitination, however, phosphorylationdependent assembly of the MRN-MDC1 complex was difficult to reconstitute, thus impeding further investigation. Moreover, we showed that overexpression of USP7 had no evident effect on the ubiquitination levels of MRE11, RAD50, or NBS1 (Supplemental Figure 4, D-F). Together, these results indicate that USP7 targets MDC1 for deubiquitination and that MDC1 is a bona fide substrate of the deubiquitinase USP7.

USP7-promoted MDC1 stabilization is potentiated by DNA damage. Since MDC1 is an essential player in the sensing and repair of DSBs, we were interested in whether genotoxic insults could have any influence on USP7-promoted stabilization of MDC1. To this end, HeLa cells were first exposed to IR, and the cellular extracts were collected at different time points. Remarkably, the protein, but not mRNA, levels of MDC1 increased at earlier time points after IR treatment (Figure 5, A and B). Likewise, the radiomimetic DNA damage agent neocarzinostatin (NCS) also resulted in elevated protein levels of MDC1 (Figure 5, A and B). Notably, we found that IR-induced MDC1 upregulation was abrogated upon USP7 depletion (Figure 5C). These results indicated that the abundance of MDC1 is regulated by USP7 upon exposure of cells to DNA damage. To support this observation, we found that the interaction between USP7 and the MRN-MDC1 complex was markedly enhanced upon IR treatment (Figure 5D). UV or mitomycin C (MMC) treatment also showed similar effects (Figure $5 \mathrm{E}$ ). These results suggest that the physical association between USP7 and the MRN-MDC1 complex is augmented by DNA damage.

Next, we tested whether USP7 acts to remove MDC1 polyubiquitination chains upon DNA damage. To this end, HeLa cells stably expressing FLAG-MDC1 were cotransfected with HA-Ub/ WT and USP7 siRNA or Myc-USP7 followed by IR exposure. IP of cellular lysates with anti-FLAG followed by IB with anti-HA showed that IR exposure resulted in decreased levels of polyubiquitinated MDC1 species, and this effect was compromised upon USP7 knockdown (Figure 5F, left) and enhanced upon USP7 overexpression (Figure 5F, right). Together, these experiments indicate that the physical interaction and functional connection between USP7 and MDC1 are strengthened by DNA damage.

Since a series of hierarchically organized protein phosphorylation events is the principal feature that fuels the genome surveillance in the DDR (35), we hypothesized that a damageinduced increase in the binding affinity of USP7 to the MRNMDC1 complex could be phosphorylation dependent. To test this idea, we exposed cells stably expressing FLAG-USP7 to IR and purified FLAG-USP7 in the presence of phosphatase inhibitors. Mass spectrometric analysis revealed that S18, S49, T54, and S963 are potential phosphorylation sites of USP7 (Supplemental Figure 5A and Supplemental File 2). However, none of these mutants with each of these residues replaced by alanine was able to interrupt the enhanced association of USP7 with the MRN-MDC1 complex under DNA damage (Supplemental Figure 5B). Taking this with our observations that neither the phospho-binding module forkhead-associated (FHA) nor the BRCT of NBS1 and/or MDC1 is involved in the association of the MRN-MDC1 complex with USP7, we infer that this augmentation is probably not dependent on phosphorylation-mediated protein-protein interaction. Whether other types of posttranslational modifications like poly ADP-ribosylation (PARylation) or ubiquitination might be involved in this process and how the assembly of the USP7-MRNMDC1 protein complex is spatially and temporally regulated remain to be determined in the future.

MDC1 is an essential mediator in the USP7-regulated DDR. To further support the functional significance of USP7-promoted MDC1 stabilization, we next asked whether USP7 could influence the recruitment of the MRN-MDC1 complex to the break compartment. 4-OHT-driven AsiSI endonuclease experiments demonstrated that, while MDC1 and USP7 displayed colocalized 
A
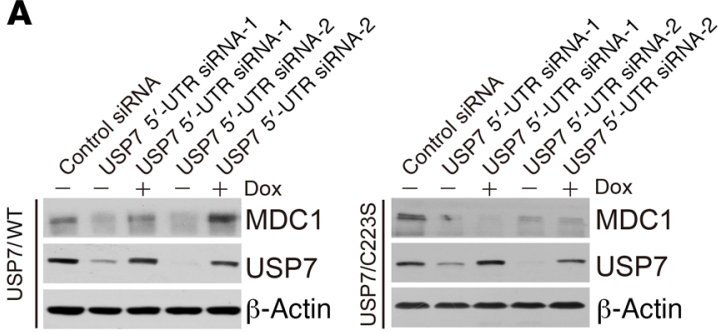

D

C

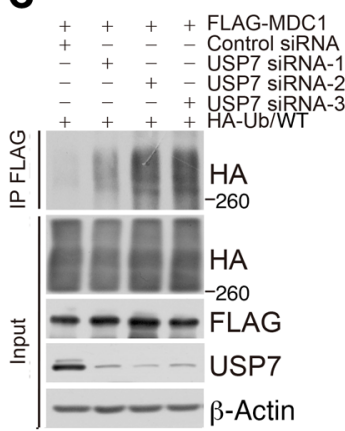

G + + + GFP-MDC

$-\quad+\quad-$ FLAG-USP7/WT

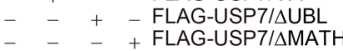
$++++\mathrm{HA}-\mathrm{Ub} / \mathrm{W} T$

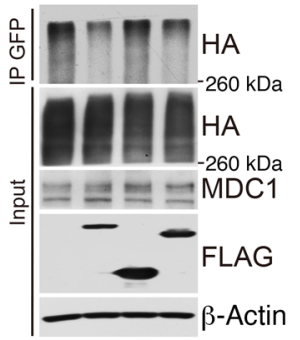

I

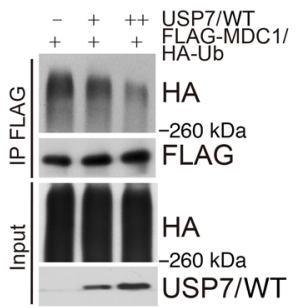

H
B

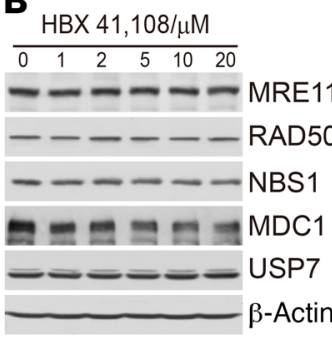

E
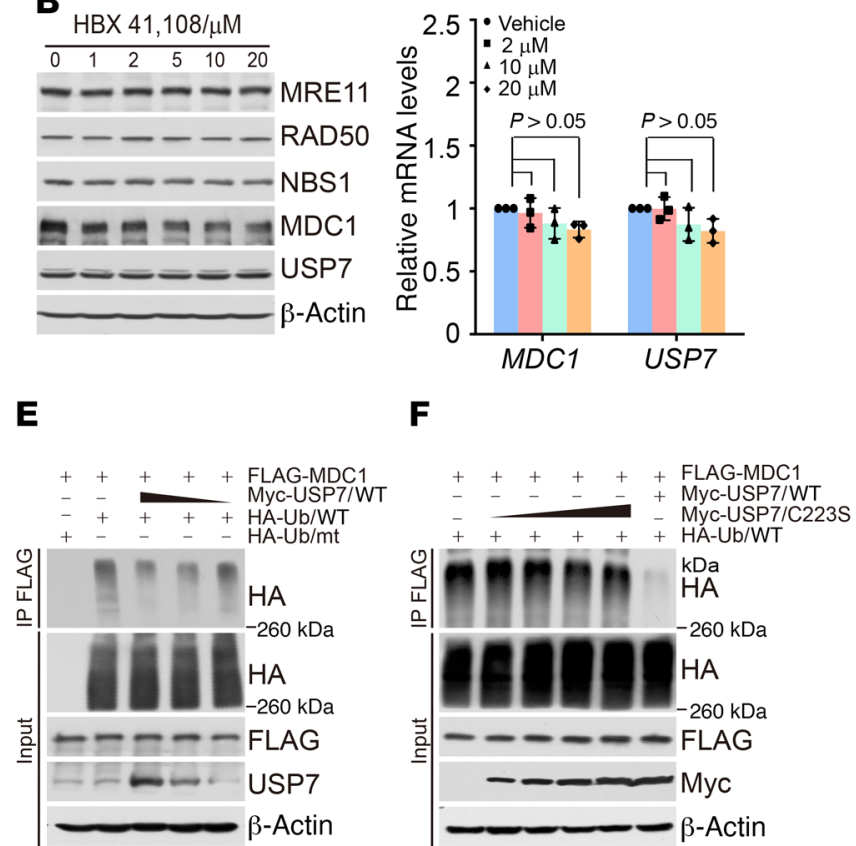

$\mathbf{F}$
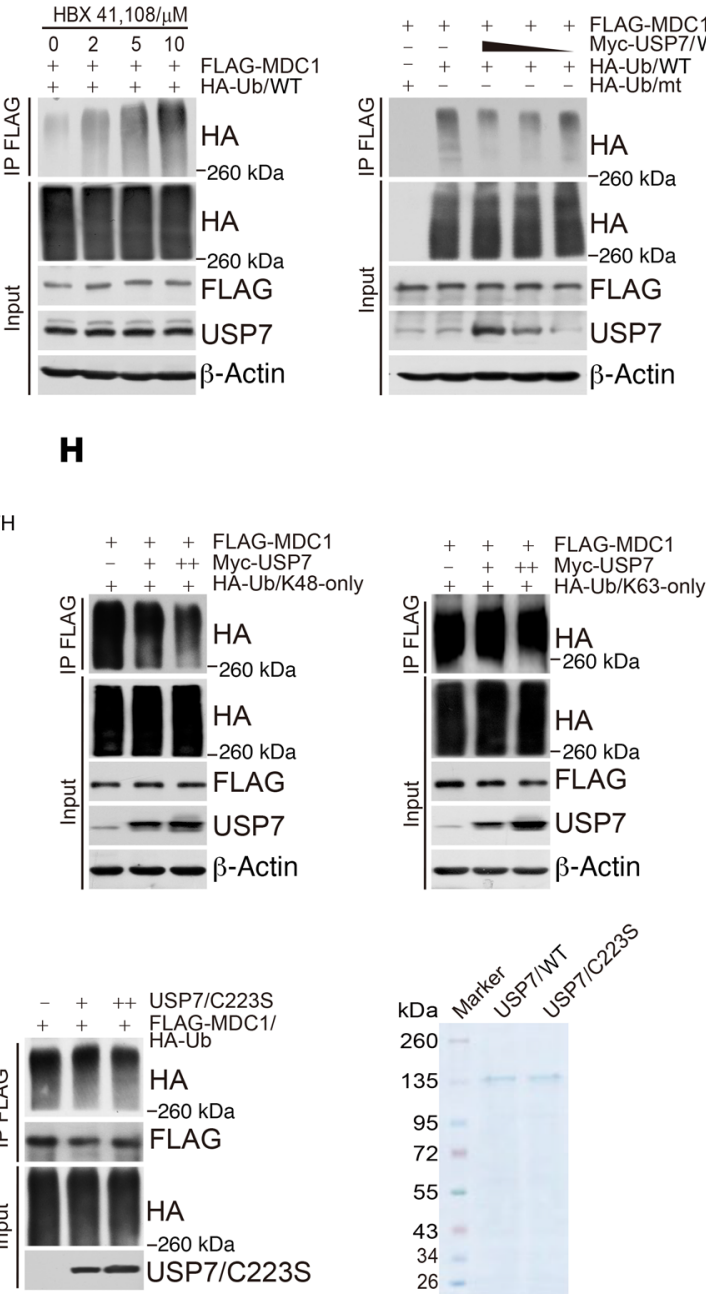

Figure 4. USP7 deubiquitinates MDC1. (A) HeLa cells with Dox-inducible expression of FLAG-USP7/WT or FLAG-USP7/C223S were transfected with control siRNA or different sets of USP7 5'-UTR siRNAs in the absence or presence of Dox. Cellular extracts were collected and analyzed by Western blotting. (B) HeLa cells were cultured in the absence or presence of increasing amounts of HBX 41,108 for 2 hours as indicated. Cellular extracts and total RNA were collected for Western blot and qRT-PCR analysis, respectively. Data represent the mean \pm SD of biological triplicate experiments. $P$ values were calculated by 1-way ANOVA. (C) HeLa cells stably expressing FLAG-MDC1 were cotransfected with control siRNA or USP7 siRNAs and HA-Ub/WT as indicated. Cellular extracts were immunoprecipitated with anti-FLAG followed by IB with anti-HA antibody. (D) HeLa cells stably expressing FLAG-MDC1 were transfected with HA-Ub/WT and cultured in the presence or absence of HBX 41,108. Cellular extracts were immunoprecipitated with anti-FLAC followed by IB with anti-HA antibody. (E) HeLa cells stably expressing FLAG-MDC1 were cotransfected with HA-Ub/WT or HA-Ub/mt and different amounts of Myc-USP7/ WT. Cellular extracts were immunoprecipitated with anti-FLAG followed by IB with anti-HA antibody. (F) HeLa cells stably expressing FLAG-MDC1 were cotransfected with HA-Ub/WT and Myc-USP7/WT or different amounts of Myc-USP7/C223S as indicated. Cellular extracts were immunoprecipitated with anti-FLAG antibody followed by IB with anti-HA antibody. (C) HeLa cells stably expressing GFP-MDC1 were cotransfected with HA-Ub/WT and FLAG-

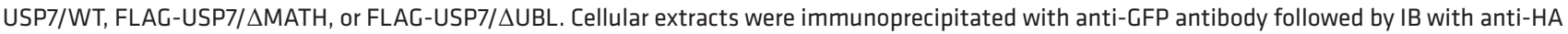
antibody. (H) HeLa cells stably expressing FLAG-MDC1 were cotransfected with different amounts of Myc-USP7/WT and HA-Ub/K48-only or HA-Ub/K63 only followed by IP analysis. (I) In vitro deubiquitination assays were performed with HA-Ub-conjugated MDC1 purified from HeLa cells using high-salt and detergent buffer and USP7/WT or USP7/C223S purified from baculovirus-infected insect cells. 
A

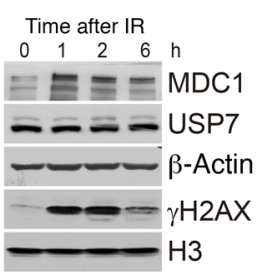

D

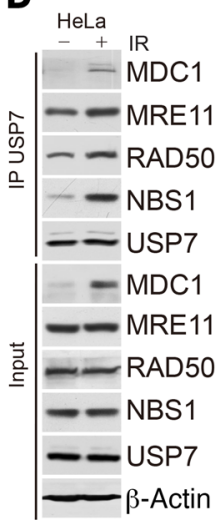

Time after NCS

$\begin{array}{lllll}0 & 1 & 2 & 6 & \mathrm{~h}\end{array}$
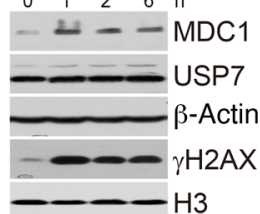

B

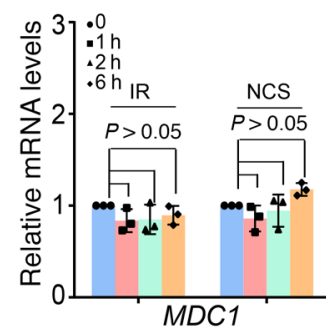

C

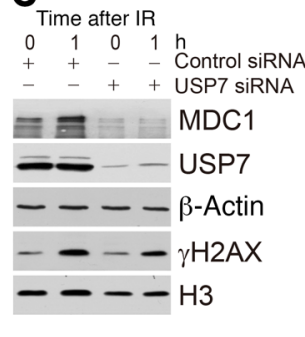

$\mathbf{E}$

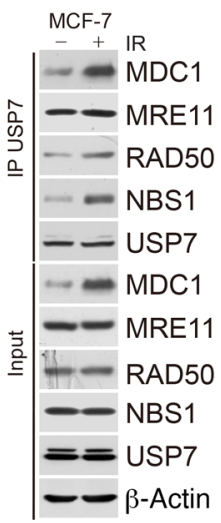

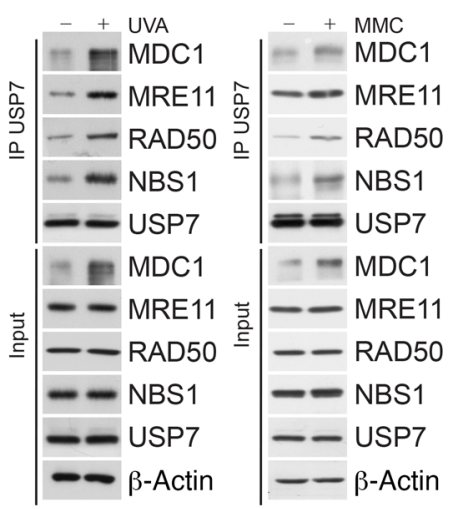

$\mathbf{F}$

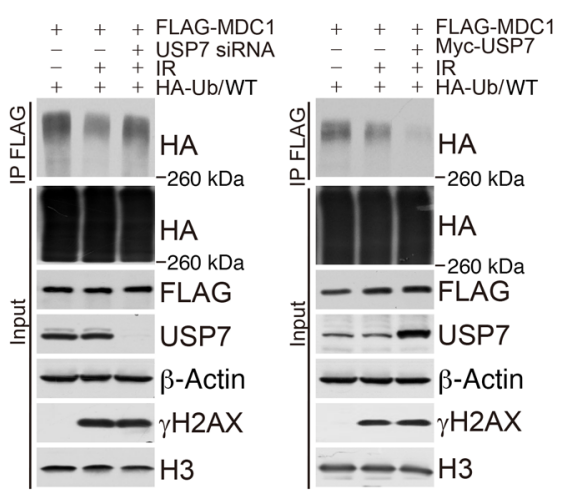

Figure 5. USP7-promoted MDC1 stabilization is potentiated by DNA damage. (A) HeLa cells exposed to IR (6 Gy) or NCS (0.5 $\mu$ g/mI) were collected at the indicated time points and analyzed by Western blotting with $\gamma \mathrm{H} 2 \mathrm{AX}$ as a positive control. (B) Total RNA was collected from HeLa cells exposed to IR or NCS followed by qRT-PCR analysis of MDC1 expression. Data represent the mean \pm SD of biological triplicate experiments. $P$ values were calculated by 1 -way ANOVA. (C) HeLa cells transfected with control siRNA or USP7 siRNA were exposed to IR and collected at the indicated time points. Expression of the indicated proteins was assessed by Western blotting. (D) HeLa cells or MCF-7 cells were exposed to IR. One hour after IR, cellular extracts were immunoprecipitated and then immunoblotted with antibodies against the indicated proteins. (E) HeLa cells were exposed to UVA (800 kJ/m²) or cultured in the alkylating agent MMC $(0.5 \mu \mathrm{g} / \mathrm{ml}, 2 \mathrm{~h})$. Cellular extracts were immunoprecipitated and then immunoblotted with antibodies against the indicated proteins. (F) HeLa cells stably expressing FLAG-MDC1 were cotransfected with HA-Ub/WT and USP7 siRNA or Myc-USP7 followed by IR treatment. Cellular extracts were immunoprecipitated with anti-FLAG antibody and then immunoblotted with anti-HA antibody.

foci in USP7-proficient cells, USP7 deficiency led to a dramatic loss of MDC1 foci formation at DSB sites (Figure 6A). Meanwhile, microscopy and live-cell imaging analysis indicated that USP7 depletion resulted in impaired recruitment of GFP-tagged MRE11, RAD50, or NBS1 at DSB sites, the effect of which could be largely reverted by MDC1 overexpression (Figure 6B and Supplemental Figure 6A; Figure 6C and Supplemental Figure 6B; and Figure 6D, and Supplemental Figure $6 \mathrm{C}$ ). We assessed protein expression by Western blotting (Supplemental Figure 6, A-C). Taken together, these observations suggest that USP7-promoted MDC1 stabilization is required for efficient accumulation of the MRN-MDC1 complex and thus the cellular response to DNA damage.

To further understand the role of USP7-promoted MDC1 stabilization in signal transduction of the DDR, we examined the effect of USP7 on foci formation of BRCA1 and 53BP1, both of which act downstream of the MRN-MDC1 complex and play key roles in DDR signaling (51-53). Consistent with the impairment of MRN-MDC1 complex accumulation in USP7-depleted cells, the number of either BRCA1 or 53BP1 foci dramatically decreased upon USP7 knockdown (Figure 6, E and F), the effect of which could be largely restored by MDC1 overexpression, albeit to a variable extent for BRCA1 and 53BP1, whereas overexpression of the E3 ligase RNF168 (Figure 6, E and F), a key regulator that bridges MDC1 and BRCA1/53BP1 through ubiquitin-dependent DNA damage signaling (18), showed a limited effect on the restoration of BRCA1 and 53BP1 foci formation. We assessed protein expression levels by Western blotting (Figure 6G).

Given that RNF168 is also reported to be a substrate of USP7 and stabilized by USP7 (40), it seems impossible that MDC1 would be able to restore the USP7 depletion-associated effect in conditions of low abundance of RNF168. To address this, we transfected control or HeLa cells stably expressing MDC1 with USP7 siRNAs. Western blot analysis showed, surprisingly, that RNF168 downregulation resulting from USP7 depletion could be reverted by MDC1 overexpression (Supplemental Figure 7A). Consistent with this finding, MDC1 knockdown led to the downregulation of RNF168 at the protein, but not mRNA, level (Supplemental Figure 7B). These observations suggest that MDC1 might function to facilitate USP7-promoted RNF168 stabilization or protect it from degradation. Although how MDC1 affects RNF168 stabilization remains to be elucidated, our results provide at least a partial explanation for how MDC1 might rescue USP7 depletion-associated DDR defects.

Previous report also suggested that USP7 acts to remove histone H2B lysine 120 monoubiquitination (H2BK12Oub1) (54). Therefore, it is possible that USP7 coordinates the response to and repair of DNA damage through deubiquitination of H2BK12Oub1. To test this hypothesis, we subjected HeLa cells with Doxinducible expression of FLAG-USP7 to UVA laser-induced DNA 
A

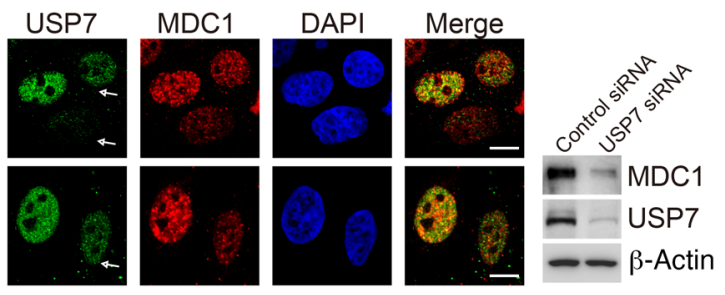

B

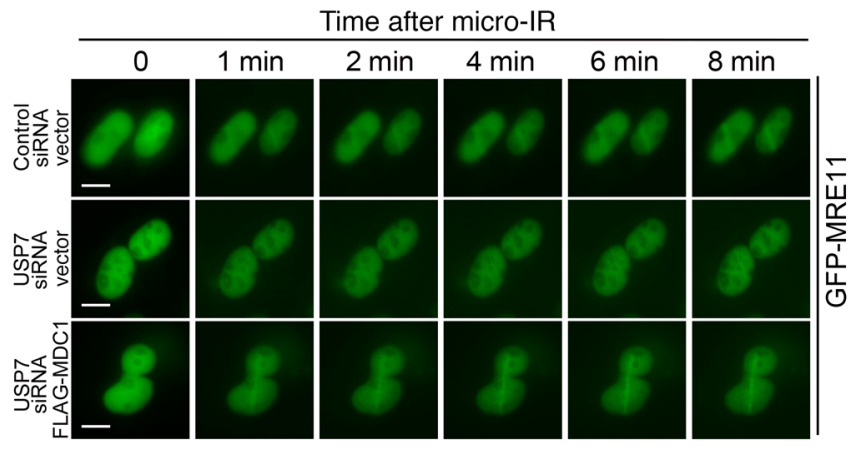

C

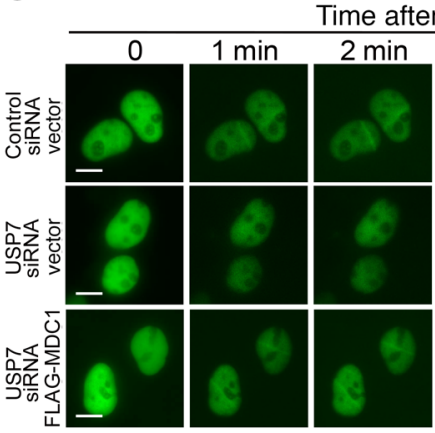

E

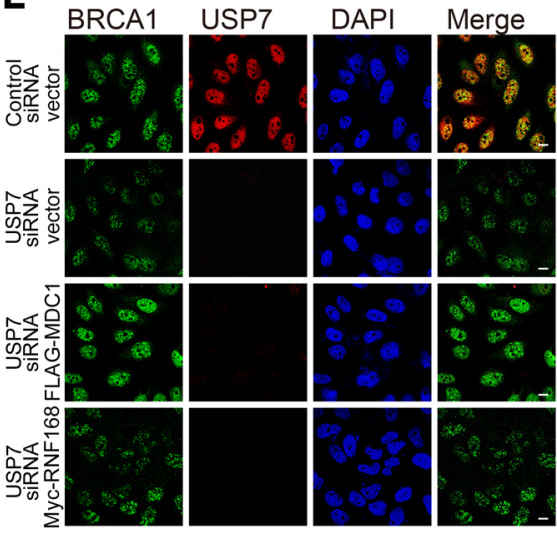

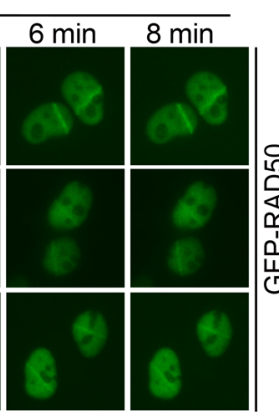

D

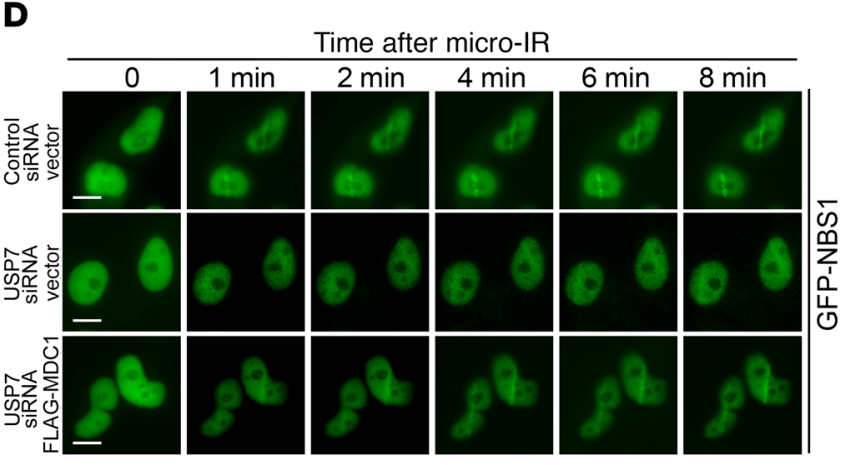

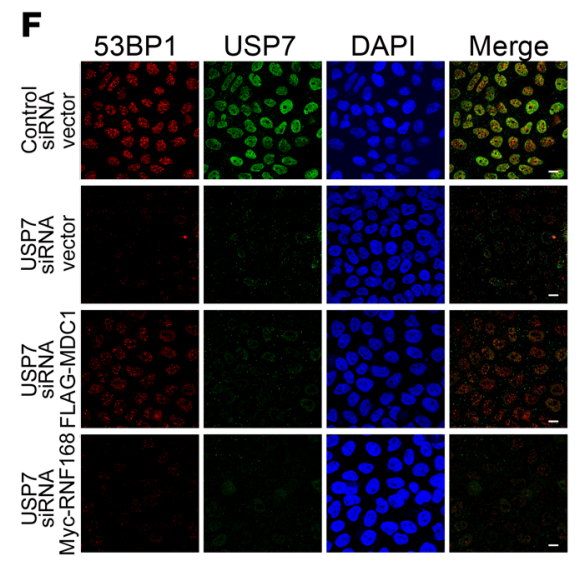

G

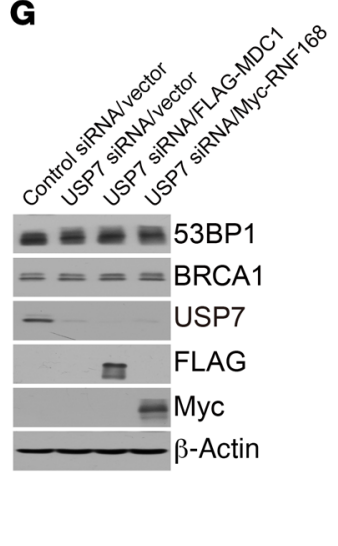

Figure 6. MDC1 is an essential mediator in the USP7-regulated DDR. (A) HeLa cells stably expressing HA-ER-AsiSI were transfected with USP7 siRNA and cultured in the presence of $0.5 \mu \mathrm{M} 4-\mathrm{OHT}$ for 4 hours. Then, cells were fixed and immunostained with the indicated antibodies followed by confocal microscopic analysis. White arrows indicate cells with different amounts of USP7 depletion. Representative images from biological triplicate experiments are shown. Scale bars: $10 \mu \mathrm{m}$. (B) HeLa cells stably expressing control vector or FLAG-MDC1 were cotransfected with the indicated siRNAs and GFP-MRE11. Then, cells were subjected to UV laser micro-IR and live-cell imaging at the indicated time points. Representative images from biological triplicate experiments are shown. Scale bars: $10 \mu \mathrm{m}$. (C) Images shown are analogous to those in B, but for GFP-RAD50. Scale bars: $10 \mu \mathrm{m}$. (D) Images shown are analogous to those B, but for GFP-NBS1. Scale bars: $10 \mu \mathrm{m}$. (E) HeLa cells stably expressing HA-ER-AsiSI were transfected with the indicated siRNAs and control vector, FLAG-MDC1, or Myc-RNF168. Cells were then treated with $0.5 \mu \mathrm{M} 4-\mathrm{OHT}$, fixed, and immunostained with antibodies against BRCA1 and USP7 followed by confocal microscopic analysis. Representative images from biological triplicate experiments are shown. Scale bars: $10 \mu \mathrm{m}$. (F) Images shown are analogous to those in E, but for 53BP1 and USP7. Scale bars: $10 \mu \mathrm{m}$. (G) Cellular extracts were collected from cells used in $\mathbf{E}$ and $\mathbf{F}$, and expression of the indicated proteins was assessed by Western blotting.

lesions. Immunofluorescence staining and confocal microscopic analysis indicated that, while the levels of H2BK12Oub1 increased at break sites as reported previously (55), USP7 overexpression had a marginal effect on this mark in laser stripe formation (Supplemental Figure 7C), although the amount of bulk monoubiquitinated H2BK120 was reduced, as determined by Western blotting (Supplemental Figure 7C), suggesting that H2BK120ub1 is not a relevant substrate of USP7 at DNA lesions.
To address the biological significance of USP7-promoted MDC1 stabilization in the DDR, we next studied how a defect in MDC1 stabilization would affect the repair of DSBs. Using a reporter assay for HR, we found that USP7 depletion resulted in compromised HR and that this effect could be largely restored by forced expression of MDC1, but not BRCA1 (Supplemental Figure 7D). Analogously, we found that MDC1 overexpression was able to counteract non-homologous end-joining (NHEJ) defects associ- 
A

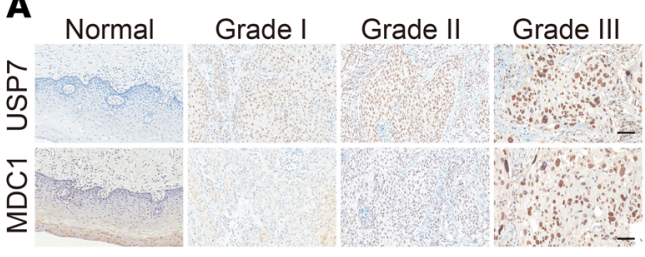

B
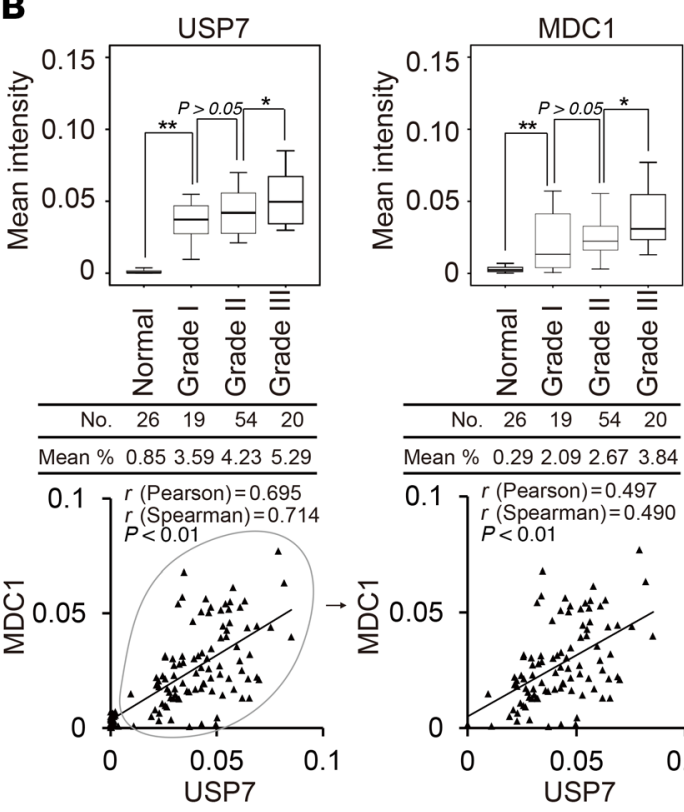

Mean \% 0.292 .092 .673 .84

$0.1 r$ (Pearson $)=0.497$

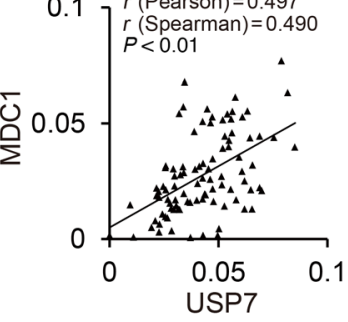

D

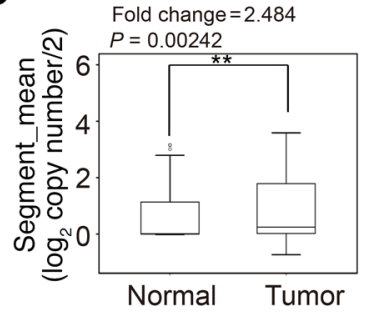

Normal (paracancerous tissue, 158 samples) Tumor (cervical squamous cell carcinoma and adenocarcinoma, 167 samples)
E

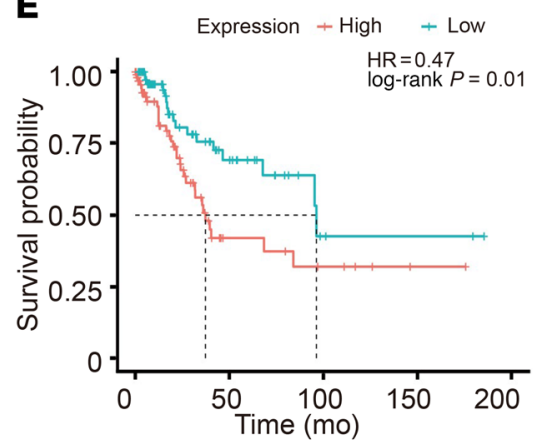

Number at risk

$\begin{array}{lcccc}\text { High113 } & 9 & 5 & 1 & 0 \\ \text { Low } 91 & 20 & 3 & 2 & 0\end{array}$

C
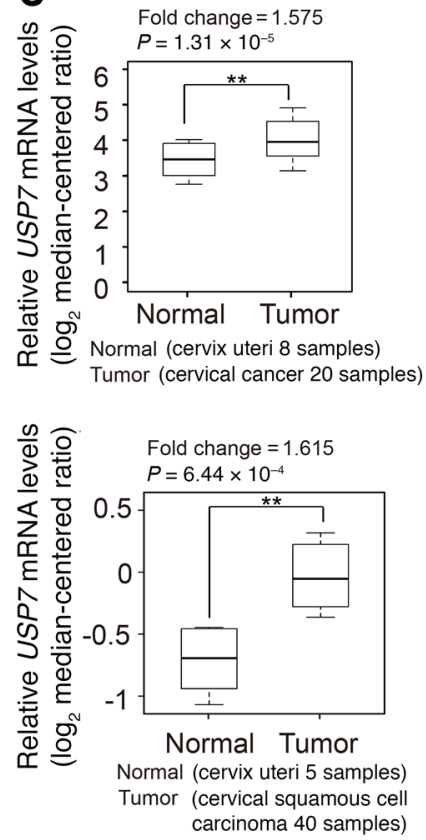

Figure 7. USP7 is implicated in cervical carcinogenesis and patient survival. (A) Human tissues containing cervical carcinoma with different grades and tumor-adjacent cervical samples were analyzed by IHC staining. Representative images (original magnification, $\times 200$ ) are shown. Scale bars: $50 \mu \mathrm{m}$. (B) Values for the stainings from A were determined by ImagePro Plus software and are presented with box plots. Boxes represent the 25th and 75th percentiles, lines represent the median, and whiskers show the minimum and maximum points. ${ }^{*} P<0.05$ and ${ }^{* *} P<0.01$, by 1 -way ANOVA. The correlation coefficient and $P$ values were analyzed as indicated. (C) Analysis of Pyeon cervix (left) or Biewenga cervix (right) from Oncomine for the expression of USP7 in normal human cervical tissues and cervical carcinoma samples. Data are presented with box plots (**P<0.01, by unpaired, 2 -tailed Student's $t$ test). (D) Bioinformatics analysis of TCGA data set (downloaded from https://portal.gdc.cancer.gov/projects/TCGA-CESC) for the gene copy numbers of USP7 in cervical paracancerous tissue and cervical carcinoma samples. Data are presented with box plots $\left({ }^{* *} P<0.01\right.$, by unpaired, 2-tailed Student's $t$ test). (E) Kaplan-Meier survival analysis for the relationship between the survival cervical cancer patients and expression levels of USP7 mRNA, with survival packages from TCFA (https://tcga-data.nci.nih.gov/docs/publications/tcga/). (F) Kaplan-Meier survival analysis of the relationship between the survival of cervical cancer patients and gene copy number status of USP7, with survival packages from TCGA (https://tcga-data.nci.nih.gov/docs/publications/tcga/).

ated with USP7 knockdown, while 53BP1 overexpression failed to do so (Supplemental Figure 7E). These findings are in agreement with the role of MDC1 in promoting both HR and NHEJ $(56,57)$. Meanwhile, comet assays showed that USP7 depletion led to significantly more residual damage after IR exposure, as evidenced by the increase in comet tail lengths, and that MDC1 overexpression decreased tail formations in the USP7-deficient cells (Supplemental Figure 7F). Consistent with the role of MDC1 in regulating the $\mathrm{G}_{2} / \mathrm{M}$ cell-cycle checkpoint in response to DNA damage (14, 21), we found that, after IR exposure, USP7 knockdown resulted 
in an increase in cells with histone $\mathrm{H} 3$ phosphorylation on serine 10 (p-H3 [S10]) and that this checkpoint defect could be reverted, to a certain extent, by MDC1 overexpression (Supplemental Figure 7G). In line with these findings, USP7 depletion significantly compromised cell survival after IR or camptothecin (CPT) treatment (Supplemental Figure 7H), and forced expression of MDC1 was capable of reducing the cellular sensitivity associated with USP7 knockdown (Supplemental Figure 7H). Collectively, these data support the notion that MDC1 is a critical mediator in the USP7-regulated DDR process.

USP7 is implicated in cervical carcinogenesis and patient survival. In light of the observations that dysregulation of the DDR is involved in promoting tumorigenesis (53) and of the implication of USP7 in the development and progression of multiple malignancies (58-60), it is reasonable to postulate that USP7-promoted MDC1 stabilization plays a role in tumorigenesis. To this end, we first analyzed USP7 and MDC1 protein levels in human tissues including in series of tumor samples from stomach, liver, pancreas, prostate, cervix, lung, ovary, rectum, kidney, and colon, with each type of cancer having 3 paired samples of adjacent normal tissue. IHC staining showed an upregulation of USP7 and MDC1 in carcinomas derived from ovary and cervix in at least 2 of the 3 paired samples (Supplemental Figure 8). To exclude the possibility that the observed IHC signal was derived from antibody nonspecific binding, the tissue sections were stained with isotype IgG or antibodies against USP7 or MDC1 (Supplemental Figure 9A).

Since cervical cancer is a type of malignancy that has a defective $G_{1} / S$-phase checkpoint stemming from mutational or functional inactivation of p53 or retinoblastoma protein $(\mathrm{pRb})$ and displays an addiction to repairing proteins to correct replication- or damage-induced DNA lesions (61), we next performed IHC stainings to analyze the expression profiles of USP7 and MDC1 in samples from cervical carcinomas of different grades and histologically normal cervix tissues in tumor-adjacent regions (Figure 7A). Quantitation analysis of the stainings showed that USP7 and MDC1 were highly expressed in cervical carcinoma samples and that their expression levels positively correlated with each other and the histological grades of cervical cancer (Figure 7B). In addition, analysis of the integrated cancer microarray database Oncomine (62) revealed that USP7 mRNA levels are markedly upregulated in cervical carcinoma samples (Figure 7C), and copy number variation (CNV) analysis of The Cancer Genome Atlas (TCGA) data set revealed that USP7 is amplified in cervical cancer (Figure 7D). Importantly, Kaplan-Meier survival analysis from TCGA data sets indicates that either elevated USP7 expression or increased copy numbers positively correlates with poor survival in patients with cervical carcinoma (Figure 7, E and F). Together, these findings point to a tumor-promoting role for USP7 in cervical cancer.

Next, we examined IHC stainings for expression of USP7 in genital warts and cervical intraepithelial neoplasia (CIN) lesions. The results indicated that expressions level of USP7 were upregulated in CINs, while limited changes were detected in genital warts (Supplemental Figure 9B). Since low-risk HPVs are most frequently detected in genital warts and high-risk HPVs exist in a substantial proportion of precancerous lesions and cervical cancer $(29,63)$, we speculate that the upregulation of USP7 is potentially linked to high-risk HPV infection in CINs and cervical cancer.
In parallel with USP7, we also examined the expression profile of MDC1 in genital warts and CINs, and the results showed that its expression level correlates with that of USP7 (Supplemental Figure 9B), suggesting that USP7-promoted MDC1 stabilization is potentially associated with cervical carcinogenesis.

To further understand whether HPV is a causal agent of the elevated expression of USP7 in cervical cancer, we tested whether HPV oncoproteins are able to drive USP7 upregulation. However, neither overexpression of E1, E2, E6, or E7 from HPV-11 (low-risk HPV), HPV-16 (high-risk HPV), or HPV-18 (high-risk HPV) in HPVnegative cervical cancer cells, nor knockdown of E6 or E7 of HPV16 or HPV-18 in HPV-positive cervical cancer cells had a detectable effect on the expression of USP7 (Supplemental Figure 9, C and D), suggesting that, unlike $\mathrm{p} 53$ or $\mathrm{pRb}$, the upregulation of USP7 could not be directly attributed to these oncogenic HPV proteins.

Considering that high-risk HPV infection and integration could result in chromosomal rearrangements and genomic instability through either inactivating checkpoint proteins or promotion of DNA re-replication of infected cells $(29,64,65)$, we hypothesized that dysregulated USP7 could be a consequence of persistent highrisk HPV infection-induced genome amplification. To test this idea, we performed DNA FISH assays with a USP7 FISH probe (16p13.2) to determine the copy numbers of USP7 in normal human cervical epithelial cells (HCerEpiC) and cervical cancer cells including C33A (HPV-negative), DoTc2 4510 (HPV-negative), SiHa (HPV-16positive), CaSki (HPV-16- and HPV-18-positive), HeLa (HPV-18positive), and MS-751 (HPV-18- and HPV-45-positive) cells. Confocal microscopic analysis showed that high-risk HPV-positive cells, but not normal cervix cells or HPV-negative cells, had an overt gain of USP7 copies (Supplemental Figure 9E). Normalization with the signal from chromosome 16 centromeric probe (CCP16) hybridizing to the same cell indicated that the increase in USP7-hybridizing loci was not due to polyploidy of chromosome 16 (Supplemental Figure 9E). Meanwhile, we demonstrated that expression levels of USP7 were substantially elevated in HPV-infected cancer cells, while no evident changes were observed in HPV-negative cancer cells (Supplemental Figure 9F). We also showed that the expression profile of MDC1 in these cells had a pattern similar to that of USP7. These observations suggest that high-risk HPV infection is possibly associated with the acquisition of extra USP7 copies and that the elevated expression of USP7 in HPVpositive tumors is probably a result of USP7 amplification.

In support of the argument that the amplification of USP7 contributes to its upregulation, CNV analysis of a data set from TCGA revealed that USP7 is amplified in cervical cancer (Figure 7D) and that its copy numbers correlate with mRNA expression levels (Supplemental Figure 9G). Collectively, we propose that a gain of USP7 copy numbers is at least one of the candidate drivers that induces USP7 upregulation in cervical cancer, although the causal link between HPV infection and USP7 amplification or its elevated expression remains to be established.

USP7 promotes cervical carcinogenesis through stabilization of MDC1. To test whether USP7-promoted MDC1 stabilization is linked to cervical carcinogenesis, we developed cervical cancer cells with stable depletion of USP7 by specific shRNAs. Colony formation assays showed that knockdown of USP7 severely impeded the colony formation of HeLa cells (cervical adenocarcinoma) and that overexpression of MDC1 could offset the 
A

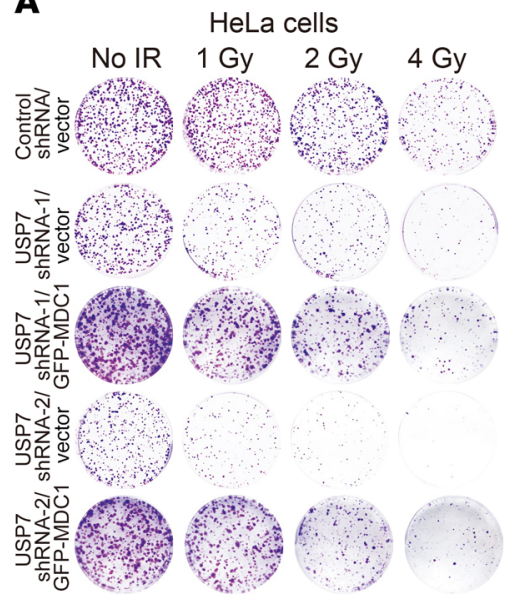

B

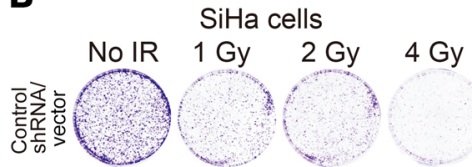

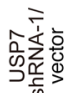

敘号

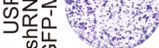

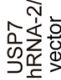

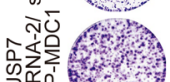

C

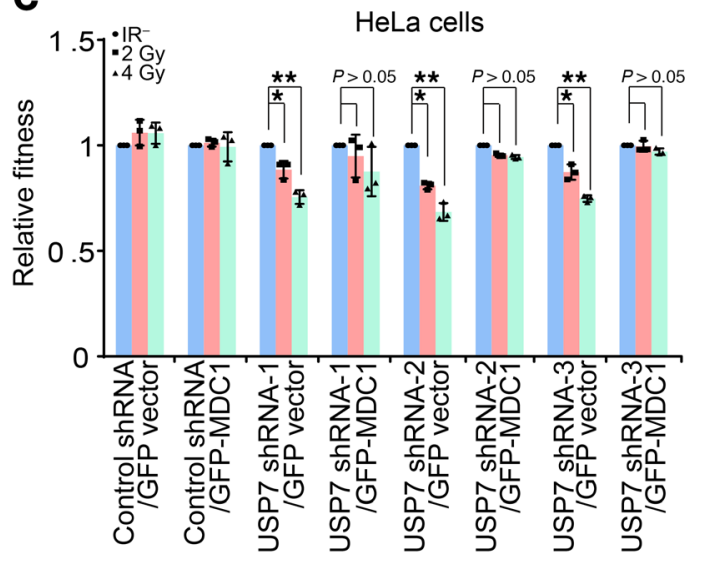

D

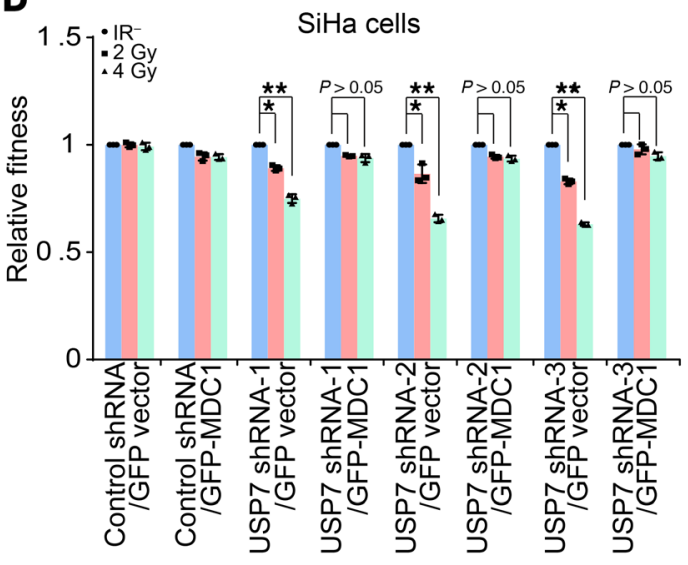

E
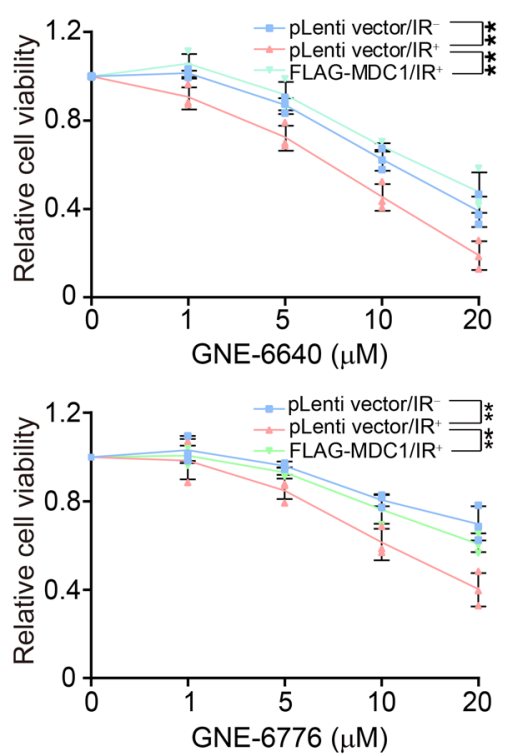

$\mathbf{F}$
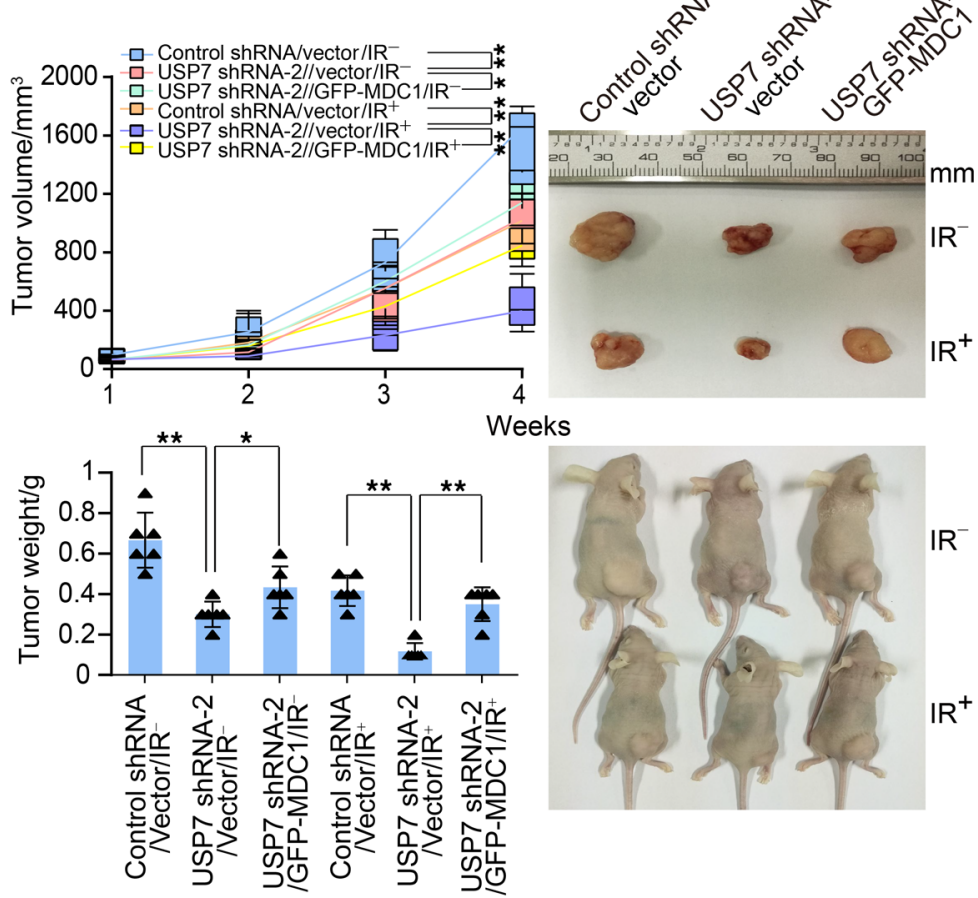
Figure 8. USP7-promoted MDC1 stabilization is required for the growth of cervical cancer cells. (A) HeLa cells stably expressing the indicated shRNAs and genes were irradiated with different doses of $x$-ray IR. Colony formation assays were conducted, and representative images from biological triplicate experiments are shown. (B) Experiments analogous to those for $\mathbf{A}$ were performed using SiHa cells. Representative images from biological triplicate experiments are shown. (C) HeLa cells stably expressing the indicated shRNAs and GFP vector or GFP-MDC1 were cocultured with equivalent native HeLa cells. The mixed cells were then irradiated with different doses of $x$-ray IR and analyzed by FACS 10 days later. The percentage of GFP-positive cells relative to GFP-negative cells under IR treatment was normalized to percentages in the untreated control mixture. The ratio reflects cellular fitness. Data represent the mean \pm SD from biological triplicate experiments. ${ }^{*} P<0.05$ and ${ }^{*} P<0.01$, by 1-way ANOVA. (D) Experiments analogous to those for $\mathbf{C}$ were performed using SiHa cells. Data represent the mean \pm SD from biological triplicate experiments. ${ }^{*} P<0.05$ and ${ }^{* *} P<0.01$, by 1 -way ANOVA. (E) Cell viability analysis was performed with SiHa cells stably expressing vector or FLAGMDC1. Cells were cultured in increasing amounts of GNE-6640 or GNE-6776 followed by $x$-ray IR (2 Gy). Data represent the mean \pm SD from biological triplicate experiments. ${ }^{* *} P<0.01$, by 2 -way ANOVA. (F) SiHa tumors stably expressing shRNAs and the indicated genes were transplanted into athymic mice $(n=12)$, and half of the mice in each group were randomly chosen and subjected to 10 Gy $x$-ray IR 1 week after tumor transplantation. Tumor volumes were measured weekly, and tumors were harvested and weighed when mice were sacrificed. Data represent the mean $\pm S D$. ${ }^{*} P<0.05$ and ${ }^{*} P<0.01$, by 2 -way ANOVA for tumor volume analysis and 1-way ANOVA for tumor weight analysis. Images of representative tumors and sacrificed mice are shown.

growth defect induced by USP7 knockdown (Figure 8A). Further exposure of these cells to different doses of x-ray-generated IR followed by colony formation assays revealed that USP7-depleted cells were more vulnerable to IR (Figure 8A), which could be offset by MDC1 overexpression (Figure $8 \mathrm{~A}$ ). We made similar observations when conducting these experiments with SiHa cells (cervix squamous cell carcinoma) (Figure 8B), in which we consistently found that MDC1 was physically associated with and stabilized by USP7 (Supplemental Figure 10, A and B). Meanwhile, multicolor competition assays (MCAs) revealed that the cellular fitness of cells with USP7 deficiency was further compromised by IR exposure, while overexpression of MDC1 was able to desensitize USP7-deficient cells to genotoxic insults (Figure 8, C and D). Furthermore, cell viability analysis demonstrated that cervical cancer cells cultured in the presence of GNE-6640 or GNE-6776 had dose-dependent growth defects and that IR made these cells more vulnerable. Importantly, we showed that this sensitization could be ameliorated by MDC1 overexpression (Figure 8E). We obtained similar results in colony formation assays using these inhibitors (Supplemental Figure 10C).

To further establish the role of USP7-promoted MDC1 stabilization in cervical carcinogenesis and tumor sensitivity to DNA damage, we ectopically transplanted 3 types of cervical tumors developed from SiHa cells infected with control lentivirus, lentivirus carrying USP7 shRNA, or lentivirus carrying USP7 shRNA and GFP-MDC1, into athymic mice (BALB/c; Charles River Laboratories). After 1 week of inoculation, the tumors were subjected to 10 Gy IR exposure for half of the mice in each group. We then monitored tumor growth and mouse weights over the next 3 weeks. The results showed that in athymic mice that received a USP7-depleted tumor transplant, the tumor growth was greatly suppressed, and overexpression of MDC1 could alleviate, to some extent, USP7 deficiency-associated growth inhibition (Figure 8F). Notably, USP7 depletion resulted in increased sensitivity of the tumors to IR exposure, the effect of which was largely alleviated by MDC1 overexpression. Western blotting and IHC analysis of the harvested tumors indicated that MDC1 levels in USP7-depleted tumors were markedly decreased (Supplemental Figure 10D). Collectively, these findings support the notion that USP7-promoted MDC1 stabilization protects cervical tumor cells from genotoxic insults, which in turn contributes to cervical tumor survival and, eventually, cervical tumorigenesis.

\section{Discussion}

In this study, we identified the protein deubiquitinase USP7 as a key regulator of an early DDR and revealed that it plays an important role in regulating the assembly of the MRN-MDC1 complex at damage sites. We showed that USP7 physically interacts with the MRN-MDC1 complex, leading to stabilization of MDC1 and accumulation of the MRN-MDC1 complex and recruitment of 53BP1 and BRCA1 to DSBs. We found that USP7 was amplified in cervical cancer cells and that the abundance of USP7 was positively correlated with that of MDC1 in clinical tumor samples and inversely correlated with the survival of cervical cancer patients, pointing to USP7 and MDC1 as potential targets for cervical cancer therapy.

In eukaryotic cells, ubiquitination is believed to play a key role in the assembly as well as disassembly of DDR factors at break sites $(17,20,66,67)$. In these processes, it appears that both nondegradation- and degradation-associated ubiquitinations come into play. MDC1 is an early and essential mediator of the DSB response pathway. However, little is known about the factors that regulate MDC1 stability. In the current study, we identified USP7 as a bona fide protein deubiquitinase of MDC1, and, consistent with previous studies $(68,69)$, we revealed that MDC1 abundance was elevated at earlier time points after IR treatment, an effect that was dramatically impaired upon USP7 depletion. These observations favor a scheme in which USP7 plays a key regulatory role by stabilizing MDC1 at the early phase of the DDR. Specifically, the joining of USP7 in the MRN-MDC1 complex maintains the abundance of MDC1 and possibly acts as a rheostat to allow an appropriate level of MRN-MDC1 complex accumulation at sites of DNA damage. It has been reported that the C-terminal UBL domain of USP7 positively regulates its deubiquitinating activity and that this effect can be further allosterically enhanced by the metabolic enzyme GMPsynthetase (GMPS) $(54,70)$. In light of our observation that the UBL domain is responsible for the association of USP7 with MDC1, it is tempting to speculate that $\mathrm{MDC} 1$ might play a role similar to that of GMPS. Interestingly, the E3 ligase MDM2, another key substrate of USP7, is reported to be stabilized by MDC1 (71), supporting our deduction that MDC1 could possibly allosterically promote USP7 catalytic activity against other substrates or even MDC1 itself. This could explain why the expression of RNF168 is subjected to MDC1 control, even in the presence of a small amount of USP7. Another pertinent interpretation of this phenomenon is that MDC1 may shield RNF168 from degradation through competitive interaction with UBR5 and/or TRIP12, both of which are E3 ubiquitin ligases reported to target RNF168 for destabilization (72). In favor of this 
interpretation, we identified both UBR5 and TRIP12 as potential interactors of $\mathrm{MDC1}$, as revealed by mass spectrometric analysis of MDC1 interaction proteins. Consistent with the role of USP7 reported here, we and others have revealed that USP7 is involved in the DDR and repair through regulation of distinct protein substrates $(40,60,73)$. Thus, it will be worthwhile to study the molecular mechanisms that determine the selectivity and coordination of USP7 substrates in the response to and repair of DSBs.

Underlining the physiological relevance of our findings, both USP7 and MDC1 were significantly overexpressed in a subset of patients with cervical cancer, and the levels of these 2 factors positively correlated with each other. It is known that 99\% of cervical cancers are associated with HPV infection, the incorporation of which results in a defective $G_{1} / S$ checkpoint $(28,61)$. The activation of the repair machinery and $G_{2} / M$ checkpoint thus becomes essential for protecting cancer cells from both endogenous replication stress and exogenous genotoxic insults. These features enable cervical cancer to be more clinically sensitive to DNA-damaging agents. However, resistance to DNA-damaging therapy alone exists, as evidenced by the poor survival rates among patients with advanced HPVpositive cervical cancer $(28,61)$. Therefore, therapeutic modulation of the DDR is an attractive strategy to increase the response to chemotherapy or radiotherapy of patients suffering from cervical cancer. Targeting USP7 may thus provide an effective treatment for patients with cervical cancer who undergo DNA-damaging therapy, particularly in light of recent efforts to develop a specific small-molecule antagonist toward the catalytic activity of USP7 $(47,74)$ or attenuate ubiquitin binding $(48$, 75). Indeed, we reveal that GNE-6640 and GNE-6776, which preferentially compete with K48-linked ubiquitin chains for binding to USP7, destabilize MDC1, thereby rendering cervical cancer cells more vulnerable to IR. In a way, this provides direct evidence for the idea that USP7 and MDC1 are viable therapeutic targets for the treatment of cervical cancer. In support of this, a recent study indicated that USP7 inhibition sensitizes p53defective, chemotherapy-resistant chronic lymphocytic leukemia (CLL) cells to clinically achievable doses of chemotherapeutic agents in a murine xenograft model (76). Thus, USP7 could be a promising therapeutic target, at least for the treatment of malignancies with DDR defects in which the p53-dependent apoptosis pathway is compromised. In support of the pathological significance of MDC1 dysregulation as well as our current work, recent studies suggest that silencing MDC1 enhances the radiosensitivity of human nasopharyngeal cancer in xenograft models (77) and that patients with oral squamous cell carcinoma with weak expression of MDC1 protein benefit significantly when treated with surgery followed by radiation therapy (78).

Given the complex biological heterogeneity of cervical cancer, it will be important to determine to what extent USP7-promoted MDC1 stabilization affects tumor sensitivity to DNA damage across genetically distinct subgroups of cervical cancer. Also, the contribution of USP7-promoted MDC1 stabilization at the posttranscriptional level to increased MDC1 expression in cervical carcinoma remains to be investigated, since MDC1 mRNA expression levels were also reported to increase in cervical cancer tissues compared with levels in case-matched normal tissues (79).
In agreement with a protective role for MDC1 in counteracting excessive, replication-associated DNA damage $(14,69,80)$, we also demonstrated that, in the absence of exogenous DNA damage, USP7-promoted MDC1 stabilization is required for cervical cancer survival. However, we cannot exclude the possibility that the role of MDC1 in the restoration of USP7 deficiency-associated growth defects is due to molecular mechanisms other than the response to and repair of DNA damage, as MDC1 itself has been reported to play a crucial role in mitotic progression (81).

It is believed that the DDR is required for productive HPV replication $(82,83)$. However, it seems unlikely that USP7 is involved in DDR-activated virus genome amplification of low-risk HPV types in genital warts, as the expression level of USP7 in these benign lesions is similar to that in normal cervical tissue. Interestingly, we found that in CIN samples, the expression level of USP7 was elevated in the mid-and upper-epithelial layers, implying that high-risk HPVs might be a causal agent of USP7 upregulation and that, in turn, the USP7-regulated response to and repair of DNA damage may drive the overreplication of the viral genome.

The extraordinary level of substrate diversity and the magnitude of its involvement in fundamental cellular and developmental processes define USP7 as a molecule of great importance. It will also be important to investigate other mechanisms controlling USP7 dysregulation in cervical cancer and the role of USP7promoted MDC1 stabilization in cellular processes including mitosis and meiosis. Furthermore, it will be interesting to explore the relationship between substrate diversity and the distinct cellular activities of USP7. Nevertheless, our study identifies USP7 as an essential regulatory subunit of the MRN-MDC1 complex in the early DDR. Our findings indicate that USP7-promoted MDC1 stabilization is critically involved in cervical carcinogenesis and support the pursuit of these molecules as potential targets for cervical cancer intervention.

\section{Methods}

For a detailed description of all methods, see the Supplemental Methods.

siRNA, shRNA, and primer sequences. Sequences of siRNAs, shRNAs, and qPCR primers are provided in Supplemental File 3.

Immunofluorescence. Cells on glass coverslips (BD Biosciences) were fixed with $4 \%$ paraformaldehyde and permeabilized with $0.2 \%$ Triton X-100 in PBS. Samples were then blocked in 5\% donkey serum in the presence of $0.1 \%$ Triton X-100 and stained with the appropriate primary and secondary antibodies coupled to Alexa Fluor 488 or 594 (Invitrogen, Thermo Fisher Scientific). Confocal images were captured on an Olympus FluoView 1000 microscope using a $\times 100$ oil objective. To avoid bleed-through effects in double-staining experiments, each dye was scanned independently in a multitracking mode. To pre-extract nonchromatin-associated proteins, cells were treated with CSK buffer (10 mM HEPES, pH 7.0, $100 \mathrm{mM} \mathrm{NaCl,} 300 \mathrm{mM}$ sucrose, $3 \mathrm{mM} \mathrm{MgCl}_{2}, 0.7 \%$ Triton X-100) twice for 3 minutes each at room temperature before fixation.

IR and laser micro-IR. IR was delivered by an x-ray generator (RS 2000 Pro, Rad Source Technologies; 160 kV, 25 mA). Micro-IR was performed with a Leica microscope equipped with a $37^{\circ} \mathrm{C}$ heating stage and a 365-nm laser diode (Andor Technology). A laser setting of $50 \%$ of full power was chosen to generate a detectable laser path. 
Laser microdissection. Cells were grown on LabTek II chamber slides (Thermo Fisher Scientific) in the presence of phenol red-free medium (Invitrogen, Thermo Fisher Scientific) before induction of DNA damage by a UVA laser ( $\lambda=355 \mathrm{~nm}, 40 \%$ energy) using a Zeiss Observer.Z1 inverted microscope with a PALM MicroBeam laser microdissection workstation under a $\times 40$ objective lens. After IR, the cells were incubated at $37^{\circ} \mathrm{C}$ for $2,10,20$, or 30 minutes and processed for immunostaining.

Statistics. Data from biological triplicate experiments are presented, and data represent the mean \pm SD. An unpaired, 2-tailed Student's $t$ test was used for 2-group comparisons. ANOVA with Bonferroni's correction was used to compare multiple groups. Values that were less than or equal to the first quartile minus 1.5 times the interquartile range or that were greater than or equal to the third quartile plus 1.5 times the interquartile range were defined as outliers and indicated with a circle. A P value of less than 0.05 was considered statistically significant. All statistical results were determined using SPSS software (IBM). Before statistical analysis, variations within each group and the assumptions of the tests were checked.

Study approval. All procedures involving animals were approved by the ethics committee of the Tianjin Medical University and followed the NIH's Guide for the Care and Use of Laboratory Animals (8th ed., National Academies Press, 2011). All studies associated with patients' samples were approved by the ethics committee of the Tianjin Medical University, and informed consent was obtained from all patients.

\section{Author contributions}

DS, SM, and L. Shi conceived this project. DS, SM, L. Shan, Yuejiao Wang, CC, NY, NS, LL, QZ, and SY conducted experiments. DS, SM, L. Shan, Yue Wang, BL, CY, ST, and KZ acquired data. DS, SM, LW, XL, ST, KZ, XD, FY, and L. Shi analyzed data. DS, SM, and L. Shi wrote the manuscript.

\section{Acknowledgments}

This work was supported by grants from the National Natural Science Foundation of China (81722036 and 31671474, to LS and 81502408, to LL, and 81602511, to NY) and the Excellent Talent Project of Tianjin Medical University(to LS). We thank Tieshan Tang (Chinese Academy of Sciences, Beijing, China), Xingzhi Xu (Shenzhen University, Shenzhen, China), and Bin Peng (Shenzhen University, Shenzhen, China) for providing help with the laser micropoint experiments.

Address correspondence to: Lei Shi, Department of Biochemistry and Molecular Biology, Tianjin Medical University, 22 Qixiangtai Road, Tianjin 300070, China. Phone: 86.22.83336998; Email: shilei@tmu.edu.cn.
1. Curtin NJ. DNA repair dysregulation from cancer driver to therapeutic target. Nat Rev Cancer. 2012;12(12):801-817.

2. Aguilera A, Gómez-González B. Genome instability: a mechanistic view of its causes and consequences. Nat Rev Genet. 2008;9(3):204-217.

3. Jeggo PA, Pearl LH, Carr AM. DNA repair, genome stability and cancer: a historical perspective. Nat Rev Cancer. 2016;16(1):35-42.

4. Bartkova J, et al. DNA damage response as a candidate anti-cancer barrier in early human tumorigenesis. Nature. 2005;434(7035):864-870.

5. Stracker TH, Petrini JH. The MRE11 complex: starting from the ends. Nat Rev Mol Cell Biol. 2011;12(2):90-103.

6. Cherry SM, Adelman CA, Theunissen JW, Hassold TJ, Hunt PA, Petrini JH. The Mre11 complex influences DNA repair, synapsis, and crossing over in murine meiosis. Curr Biol. 2007;17(4):373-378.

7. Hopfner KP, et al. The Rad50 zinc-hook is a structure joining Mre11 complexes in DNA recombination and repair. Nature. 2002;418(6897):562-566.

8. Bhaskara V, et al. Rad50 adenylate kinase activity regulates DNA tethering by Mre11/Rad50 complexes. Mol Cell. 2007;25(5):647-661.

9. Roset R, et al. The Rad50 hook domain regulates DNA damage signaling and tumorigenesis. Genes Dev. 2014;28(5):451-462.

10. Williams RS, et al. Nbs1 flexibly tethers Ctp1 and Mre11-Rad50 to coordinate DNA doublestrand break processing and repair. Cell. 2009;139(1):87-99.

11. Lloyd J, et al. A supramodular FHA/BRCTrepeat architecture mediates Nbs1 adaptor function in response to DNA damage. Cell. 2009;139(1):100-111.
12. Spycher C, et al. Constitutive phosphorylation of MDC1 physically links the MRE11-RAD50NBS1 complex to damaged chromatin. J Cell Biol. 2008;181(2):227-240.

13. Chapman JR, Jackson SP. Phospho-dependent interactions between NBS1 and MDC1 mediate chromatin retention of the MRN complex at sites of DNA damage. EMBO Rep. 2008;9(8):795-801.

14. Stewart GS, Wang B, Bignell CR, Taylor AM, Elledge SJ. MDC1 is a mediator of the mammalian DNA damage checkpoint. Nature. 2003;421(6926):961-966.

15. Berkovich E, Monnat RJ, Kastan MB. Roles of ATM and NBS1 in chromatin structure modulation and DNA double-strand break repair. Nat Cell Biol. 2007;9(6):683-690.

16. Goldberg M, et al. MDC1 is required for the intra-S-phase DNA damage checkpoint. Nature. 2003;421(6926):952-956.

17. Mattiroli F, et al. RNF168 ubiquitinates K13-15 on $\mathrm{H} 2 \mathrm{~A} / \mathrm{H} 2 \mathrm{AX}$ to drive DNA damage signaling. Cell. 2012;150(6):1182-1195.

18. Doil C, et al. RNF168 binds and amplifies ubiquitin conjugates on damaged chromosomes to allow accumulation of repair proteins. Cell. 2009;136(3):435-446.

19. Huen MS, et al. RNF8 transduces the DNAdamage signal via histone ubiquitylation and checkpoint protein assembly. Cell. 2007;131(5):901-914.

20. Mailand N, et al. RNF8 ubiquitylates histones at DNA double-strand breaks and promotes assembly of repair proteins. Cell. 2007;131(5):887-900

21. Lou Z, et al. MDC1 maintains genomic stability by participating in the amplification of ATM-dependent DNA damage signals. Mol Cell. 2006;21(2):187-200.

22. Venkitaraman AR. Cancer susceptibility and the functions of BRCA1 and BRCA2. Cell.
2002;108(2):171-182.

23. Zhao $\mathrm{W}$, et al. BRCA1-BARD1 promotes RAD51-mediated homologous DNA pairing. Nature. 2017;550(7676):360-365.

24. Scully R, Livingston DM. In search of the tumour-suppressor functions of BRCA1 and BRCA2. Nature. 2000;408(6811):429-432.

25. Esteller M, et al. Promoter hypermethylation and BRCA1 inactivation in sporadic breast and ovarian tumors. J Natl Cancer Inst. 2000;92(7):564-569.

26. Matt S, Hofmann TG. The DNA damage-induced cell death response: a roadmap to kill cancer cells. Cell Mol Life Sci. 2016;73(15):2829-2850.

27. Reaper PM, et al. Selective killing of ATM- or p53-deficient cancer cells through inhibition of ATR. Nat Chem Biol. 2011;7(7):428-430.

28. Wieringa HW, van der Zee AG, de Vries EG, van Vugt MA. Breaking the DNA damage response to improve cervical cancer treatment. Cancer Treat Rev. 2016;42:30-40.

29. Roden RBS, Stern PL. Opportunities and challenges for human papillomavirus vaccination in cancer. Nat Rev Cancer. 2018;18(4):240-254.

30. Roossink F, et al. The role of ATM and 53BP1 as predictive markers in cervical cancer. Int $J$ Cancer. 2012;131(9):2056-2066.

31. Roychowdhury A, et al. Integrative genomic and network analysis identified novel genes associated with the development of advanced cervical squamous cell carcinoma. Biochim Biophys Acta. 2017;1861(1 Pt A):2899-2911.

32. Price BD, D'Andrea AD. Chromatin remodeling at DNA double-strand breaks. Cell. 2013;152(6):1344-1354

33. Vidanes GM, Bonilla CY, Toczyski DP. Complicated tails: histone modifications and the DNA damage response. Cell. 2005;121(7):973-976.

34. Wilson MD, et al. The structural basis of modi- 
fied nucleosome recognition by 53BP1. Nature. 2016;536(7614):100-103.

35. Dantuma NP, van Attikum H. Spatiotemporal regulation of posttranslational modifications in the DNA damage response. $E M B O J$. 2016;35(1):6-23.

36. Thorslund T, et al. Histone H1 couples initiation and amplification of ubiquitin signalling after DNA damage. Nature. 2015;527(7578):389-393.

37. Huang OW, Cochran AG. Regulation of deubiquitinase proteolytic activity. Curr Opin Struct Biol. 2013;23(6):806-811.

38. van Loosdregt J, et al. Stabilization of the transcription factor Foxp 3 by the deubiquitinase USP7 increases Treg-cell-suppressive capacity. Immunity. 2013;39(2):259-271.

39. Nicholson B, Suresh Kumar KG. The multifaceted roles of USP7: new therapeutic opportunities. Cell Biochem Biophys. 2011;60(1-2):61-68.

40. Zhu Q, Sharma N, He J, Wani G, Wani AA. USP7 deubiquitinase promotes ubiquitin-dependent DNA damage signaling by stabilizing RNF168. Cell Cycle. 2015;14(9):1413-1425.

41. An L, et al. Dual-utility NLS drives RNF169dependent DNA damage responses. Proc Natl Acad Sci U S A. 2017;114(14):E2872-E2881.

42. Zhang P, et al. ATM-mediated stabilization of ZEB1 promotes DNA damage response and radioresistance through CHK1. Nat Cell Biol. 2014;16(9):864-875.

43. Alonso-de Vega I, Martín Y, Smits VA. USP7 controls Chk1 protein stability by direct deubiquitination. Cell Cycle. 2014;13(24):3921-3926.

44. Faustrup H, Bekker-Jensen S, Bartek J, Lukas J, Mailand N. USP7 counteracts SCFbetaTrCP-but not APCCdh1-mediated proteolysis of Claspin. JCell Biol. 2009;184(1):13-19.

45. Aymard F, et al. Genome-wide mapping of longrange contacts unveils clustering of DNA double-strand breaks at damaged active genes. Nat Struct Mol Biol. 2017;24(4):353-361.

46. Daubeuf S, et al. HSV ICPO recruits USP7 to modulate TLR-mediated innate response. Blood. 2009;113(14):3264-3275.

47. Reverdy C, et al. Discovery of specific inhibitors of human USP7/HAUSP deubiquitinating enzyme. Chem Biol. 2012;19(4):467-477.

48. Kategaya L, et al. USP7 small-molecule inhibitors interfere with ubiquitin binding. Nature. 2017;550(7677):534-538.

49. Shi W, et al. Disassembly of MDC1 foci is controlled by ubiquitin-proteasomedependent degradation. J Biol Chem. 2008;283(46):31608-31616.

50. Li M, Brooks CL, Wu-Baer F, Chen D, Baer R, $\mathrm{Gu}$ W. Mono- versus polyubiquitination: differential control of $\mathrm{p} 53$ fate by Mdm2. Science. 2003;302(5652):1972-1975.

51. Bouwman P, et al. 53BP1 loss rescues BRCA1 deficiency and is associated with triple-negative and BRCA-mutated breast cancers. Nat Struct
Mol Biol. 2010;17(6):688-695.

52. Bunting SF, et al. 53BP1 inhibits homologous recombination in Brca1-deficient cells by blocking resection of DNA breaks. Cell. 2010;141(2):243-254

53. Pearl LH, Schierz AC, Ward SE, Al-Lazikani B, Pearl FM. Therapeutic opportunities within the DNA damage response. Nat Rev Cancer. 2015;15(3):166-180.

54. van der Knaap JA, et al. GMP synthetase stimulates histone $\mathrm{H} 2 \mathrm{~B}$ deubiquitylation by the epigenetic silencer USP7. Mol Cell. 2005;17(5):695-707.

55. Nakamura K, et al. Regulation of homologous recombination by RNF2O-dependent H2B ubiquitination. Mol Cell. 2011;41(5):515-528.

56. Luo K, Zhang H, Wang L, Yuan J, Lou Z. Sumoylation of MDC1 is important for proper DNA damage response. $E M B O J$. 2012;31(13):3008-3019.

57. Lee KY, Im JS, Shibata E, Dutta A. ASF1a promotes non-homologous end joining repair by facilitating phosphorylation of MDC1 by ATM at double-strand breaks. Mol Cell. 2017;68(1):61-75.e5.

58. Chauhan D, et al. A small molecule inhibitor of ubiquitin-specific protease-7 induces apoptosis in multiple myeloma cells and overcomes bortezomib resistance. Cancer Cell. 2012;22(3):345-358.

59. Epping MT, Meijer LA, Krijgsman O, Bos JL, Pandolfi PP, Bernards R. TSPYL5 suppresses p53 levels and function by physical interaction with USP7. Nat Cell Biol. 2011;13(1):102-108.

60. Wang $\mathrm{Q}$, et al. Stabilization of histone demethylase PHF 8 by USP7 promotes breast carcinogenesis. JClin Invest. 2016;126(6):2205-2220.

61. Cossar LH, Schache AG, Risk JM, Sacco JJ, Jones NJ, Lord R. Modulating the DNA damage response to improve treatment response in cervical cancer. Clin Oncol (R Coll Radiol). 2017;29(9):626-634.

62. Rhodes DR, et al. Oncomine 3.0: genes, pathways, and networks in a collection of 18,000 cancer gene expression profiles. Neoplasia. 2007;9(2):166-180.

63. Moody CA, Laimins LA. Human papillomavirus oncoproteins: pathways to transformation. Nat Rev Cancer. 2010;10(8):550-560.

64. Kadaja M, Isok-Paas H, Laos T, Ustav E, Ustav M. Mechanism of genomic instability in cells infected with the high-risk human papillomaviruses. PLoS Pathog. 2009;5(4):e1000397.

65. McBride AA, Warburton A. The role of integration in oncogenic progression of HPV-associated cancers. PLoS Pathog. 2017;13(4):e1006211.

66. Fradet-Turcotte A, et al. 53BP1 is a reader of the DNA-damage-induced H2A Lys 15 ubiquitin mark. Nature. 2013;499(7456):50-54.

67. Li Y, et al. USP13 regulates the RAP80-BRCA1 complex dependent DNA damage response. Nat Commun. 2017;8:15752.
68. Zhang D, Zaugg K, Mak TW, Elledge SJ. A role for the deubiquitinating enzyme USP28 in control of the DNA-damage response. Cell. 2006;126(3):529-542.

69. Peng A, Chen PL. NFBD1, like 53BP1, is an early and redundant transducer mediating Chk2 phosphorylation in response to DNA damage. JBiol Chem. 2003;278(11):8873-8876.

70. Faesen AC, Dirac AM, Shanmugham A, Ovaa $\mathrm{H}$, Perrakis A, Sixma TK. Mechanism of USP7/ HAUSP activation by its $\mathrm{C}$-terminal ubiquitinlike domain and allosteric regulation by GMP-synthetase. Mol Cell. 2011;44(1):147-159.

71. Inoue K, et al. NFBD1/MDC1 stabilizes oncogenic MDM2 to contribute to cell fate determination in response to DNA damage. Biochem Biophys Res Commun. 2008;371(4):829-833.

72. Gudjonsson T, et al. TRIP12 and UBR5 suppress spreading of chromatin ubiquitylation at damaged chromosomes. Cell. 2012;150(4):697-709.

73. Khoronenkova SV, Dianova II, Ternette N, Kessler BM, Parsons JL, Dianov GL. ATMdependent downregulation of USP7/HAUSP by PPM1G activates $\mathrm{p} 53$ response to DNA damage. Mol Cell. 2012;45(6):801-813.

74. Gavory G, et al. Discovery and characterization of highly potent and selective allosteric USP7 inhibitors. Nat Chem Biol. 2018;14(2):118-125.

75. Turnbull AP, et al. Molecular basis of USP7 inhibition by selective small-molecule inhibitors. Nature. 2017;550(7677):481-486.

76. Agathanggelou A, et al. USP7 inhibition alters homologous recombination repair and targets CLL cells independently of ATM/p53 functional status. Blood. 2017;130(2):156-166.

77. Wang Z, et al. Silencing NFBD1/MDC1 enhances the radiosensitivity of human nasopharyngeal cancer CNE1 cells and results in tumor growth inhibition. Cell Death Dis. 2015;6:e1849.

78. Dave JH, Vora HH, Ghosh NR, Trivedi TI. Mediator of DNA damage checkpoint protein 1 (MDC1) as a prognostic marker for patients with oral squamous cell carcinoma. JOral Pathol Med. 2017;46(4):253-258.

79. Yuan C, et al. NFBD1/MDC1 is a protein of oncogenic potential in human cervical cancer. Mol Cell Biochem. 2012;359(1-2):333-346.

80. Wang J, Gong Z, Chen J. MDC1 collaborates with TopBP1 in DNA replication checkpoint control. JCell Biol. 2011;193(2):267-273.

81. Li Z, Shao C, Kong Y, Carlock C, Ahmad N, Liu $\mathrm{X}$. DNA damage response-independent role for MDC1 in maintaining genomic stability. Mol Cell Biol. 2017;37(9):e00595-16.

82. Gautam D, Moody CA. Impact of the DNA damage response on human papillomavirus chromatin. PLoS Pathog. 2016;12(6):e1005613.

83. Moody CA, Laimins LA. Human papillomaviruses activate the ATM DNA damage pathway for viral genome amplification upon differentiation. PLoS Pathog. 2009;5(10):e1000605. 